\title{
1 A quantitative principle to understand 3D cellular connectivity in 2 epithelial tubes
}

4 Pedro Gómez-Gálvez ${ }^{1,2, \dagger}$, Pablo Vicente-Munuera ${ }^{1,2, \dagger}$, Samira Anbari ${ }^{3, \dagger}$,

5 Antonio Tagua ${ }^{1,2, \dagger}$, Carmen Gordillo-Vázquez ${ }^{1,2}$, Ana M. Palacios ${ }^{1,2}$, Antonio

6 Velasco ${ }^{1}$, Carlos Capitán-Agudo ${ }^{1}$, Clara Grima ${ }^{5}$, Valentina Annese ${ }^{1,2}$, Rafael

7 Robles $^{5}$, Alberto Márquez ${ }^{5}$, Javier Buceta ${ }^{3,4, *}$, Luis M. Escudero ${ }^{1,2, *}$

9 1: Instituto de Biomedicina de Sevilla (IBiS), Hospital Universitario Virgen del

10 Rocío/CSIC/Universidad de Sevilla and Departamento de Biología Celular, 11 Universidad de Sevilla. 41013 Seville, Spain.

12 2: Biomedical Network Research Centre on Neurodegenerative Diseases 13 (CIBERNED), Madrid, Spain.

14 3: Chemical and Biomolecular Engineering Department, Lehigh University. 15 Bethlehem, PA 18018, USA.

16 4: Bioengineering Department, Lehigh University. Bethlehem, PA 18018, 17 USA.

18 5: Departamento de Matemática Aplicada I, Universidad de Sevilla. 41012 19 Seville, Spain.

22 †: These authors contributed equally to this work.

$23 *$ : Corresponding authors 


\section{ABSTRACT}

2 Apico-basal cell intercalations (scutoids) optimize packing and energy

3 expenditure in curved epithelia. Further consequences of this new paradigm of

4 tissue packing remain uncharacterized. In particular, how scutoids modify the

$53 \mathrm{D}$ cellular connectivity is an open question. This property is crucial for

6 understanding epithelial architecture and is instrumental for regulating the

7 biological function of tissues. Here, we address this problem by means of a

8 computational model of epithelial tubes and a biophysical approach that links

9 geometrical descriptors with the energetic cost required to increase the cellular

10 connectivity. Our results predict that epithelial tubes satisfy a novel

11 quantitative principle: the "Flintstones' law". In short, cellular connectivity

12 increases with tissue thickness/curvature in a logistic way. We confirm

13 experimentally the existence of this principle using Drosophila's salivary

14 glands. Our study provides methodological advances to analyze tissue

15 packing in 3D and, more importantly, unveils a morphogenetic principle with

16 key biological consequences.

\section{KEYWORDS}

18 Epithelial architecture, Tubulogenesis, Mathematical/Biophysical modeling,

19 Computational geometry, Developmental systems biology, Cellular

20 connectivity. 


\section{INTRODUCTION}

2 During the last decades much progress has been achieved in the 3 understanding of the emergence of self-organization in tissues. This problem 4 has been addressed from the viewpoint of energetics drivers (Alt et al, 2017;

5 Nelson et al, 2005; Siedlik et al, 2017; Trepat et al, 2009; Misra et al, 2017;

6 Sugimura et al, 2016; Fletcher et al, 2014; Canela-Xandri et al, 2011), 7 material-like properties (Yang et al, 2017; Bi et al, 2015; Pérez-González et al,

8 2019; Latorre et al, 2018; Mongera et al, 2018; Campàs et al, 2014), and the

9 analysis of the packing properties. As for the latter, the analysis of epithelial

10 surfaces as tessellations of convex polygons has been successfully used to

11 quantitatively understand different biological aspects such as tissue patterning,

12 cell division, and growth (Mao et al, 2013; Thompson, 1945; Farhadifar et al,

13 2007; Gibson et al, 2011, 2006; Lewis, 1928; Honda, 1978; Sánchez-Gutiérrez

14 et al, 2016; Curran et al, 2017). Importantly, these studies have also revealed

15 the validity of mathematical principles with biological consequences. One

16 relevant example are the implications of Euler's formula (Reinhardt, 1918;

17 Wetzel, 1926) about cellular connectivity. This principle states that polygonal

18 cells in packed tissues, on average, have six neighbors (i.e., the average 2D

19 cellular connectivity reads $\left\langle n_{2 D}\right\rangle=6$ ). As for its biological consequences, the

20 degree of cellular connectivity determines, for example, the strength of the

21 cell-cell juxtracrine signaling (Tung et al, 2012; Sharma et al, 2019; Perrimon

22 et al, 2012). Not surprisingly, the validity of this connectivity principle to the

23 third dimension has been taken for granted since the role played by apico-

24 basal cell intercalations has been disregarded and cells have been assumed

25 to have prismatic-like shapes in either planar or bended epithelia.

26 However, the recent discovery of more complex cellular geometries in 27 epithelial cells, i.e., scutoids, to reach an efficient three-dimensional (3D) 28 tissue packing has set a new paradigm that has not been yet fully explored 29 (Mughal et al, 2018; Gómez-Gálvez et al, 2018; Rupprecht et al, 2017).

30 Scutoids imply spatial changes in the neighboring relationship between cells

31 (Fig. 1a). This phenomenon is then a spatial version of the T1 transitions that 32 produce cell rearrangements with time in numerous developmental processes 33 (Bertet et al, 2004; Spencer et al, 2017; Irvine \& Wieschaus, 1994). Thus, the 
1 presence of scutoids necessarily modifies the connectivity and the biophysical

2 properties of tissues. Still, the analysis of tissue organization in a 3D context,

3 and the corresponding biological repercussions, have been hindered by the

4 technical difficulties to accurately segment and reconstruct cells from apical to

5 basal surfaces. In addition, very few computational models account for the

6 presence of apico-basal transitions to investigate 3D self-organization in

7 tissues (Okuda et al, 2019; Mughal et al, 2018; Gómez-Gálvez et al, 2018;

8 Rupprecht et al, 2017).

9 The realistic analysis of $3 D$ packing is in turn utterly relevant in epithelial

10 tubes, where scutoids appear more frequently (Sanchez-Corrales et al, 2018;

11 Iruela-Arispe \& Beitel, 2013; Gómez-Gálvez et al, 2018). Epithelial tubes are in

12 fact the primary developmental structures in all organisms with bilateral

13 symmetry (Gilbert \& Barresi, 2016) and tubulogenesis is fundamental in a

14 broad variety of key developmental processes, including gastrulation and

15 neurulation (Pilot \& Lecuit, 2005; Swanson \& Beitel, 2006; Colas \&

16 Schoenwolf, 2001; Leptin \& Grunewald, 1990; Nelson, 2009; Iruela-Arispe \&

17 Beitel, 2013). Furthermore, epithelial tubes are the essential functional unit of

18 many mammalian organs, including glands, components of the digestive

19 apparatus, lungs, and kidney (Huebner \& Ewald, 2014). Hence, the faithful

20 formation and function of tubes requires the precise coordination of dynamic

21 changes in the tissue architecture, i.e., packing, during development (Röper,

22 2018).

23 Here, we study the packing and the 3D cellular connectivity properties of

24 epithelial tubes. We show that the presence of scutoids implies a breakdown

25 of the principle $\left\langle n_{3 D}\right\rangle=6$ and reveal a novel law that quantitatively links the 3D

26 cellular connectivity, geometrical descriptors (e.g., tissue curvature/thickness),

27 and energetics drivers. Our findings are supported by i) a computational model

28 that realistically render the 3D cellular organization of tubular epithelia

29 (including the appearance of scutoids); ii) a biophysical model, supported by

30 mathematical calculations, that connects the tissue energetics with the 3D

31 organization of epithelial tubes; and iii) experimental data of epithelial tubes

32 (Drosophila's salivary gland) whose 3D cellular structure has been accurately

33 characterized by means of a novel computer-aided image analysis method. 
1 Altogether, by realistically capturing the organization of cells in tubular

2 epithelia, we shed light on the important issue of how tissues are 3D shaped

3 and we open the door to understand quantitatively key morphogenetic events

4 that ultimately depends on the 3D cellular connectivity.

6 RESULTS

7 A computational model unveils the connectivity properties of tubular 8 epithelia

9 To understand how the geometry of tubular epithelia affects the 3D cellular

10 packing and connectivity, we designed and implemented a computational

11 Voronoi tubular model (Gómez-Gálvez et al, 2018) (Materials and Methods).

12 We analyzed tubes with an increasing surface ratio (radial expansion),

$13 s_{b}=R_{b} / R_{a}$ (Fig. 1b). This parameter quantifies the ratio of the non-trivial

14 curvatures of apical and basal tubular surfaces, $\kappa_{a} / \kappa_{b}=R_{b} / R_{a}=s_{b}$, and it is

15 a proxy for the dimensionless tissue thickness, $\left(R_{b}-R_{a}\right) / R_{a}=\left(s_{b}-1\right)$. In

16 addition, we explored the cellular organization of tubes by using a Centroidal

17 Voronoi Tessellation (CVT) scale (Fig. 1c). The CVT scale accounts for the

18 number of iterations of the homogenizing Lloyd's algorithm and makes

19 possible to analyze the effect of the topological order of the tissue (Materials

20 and Methods) (Sánchez-Gutiérrez et al, 2016; Gómez-Gálvez et al, 2018).

21 Our results showed that the average number of apico-basal intercalations 22 per cell, $\left\langle i\left(s_{b}\right)\right\rangle$, (Fig. 1d), and therefore the percentage of cells adopting the

23 scutoidal shape (Fig. S1), increases with $s_{b}$ and decreases as tubes become

24 more ordered (i.e., as the CVT index increases). To further uncover the 3D

25 organization of tissues, we implemented a benchmark able to reveal

26 simultaneously the existence of apico-basal intercalations (scutoids) and the

27 polygonal distributions of cells. To that end, we computed the probability that

28 cells change their polygonal class between the apical and basal surfaces.

29 Thus, the components (i.e., bins) of this distribution along the diagonal

30 account for prismatic cells (Fig. 2a) whereas the spreading away from the

31 diagonal reveals the existence of scutoids (cells that exchange neighbors due

32 to apico-basal intercalations) and, consequently, changes in the cellular 3D

33 connectivity in the tissue (Fig. 2b). In agreement with the results shown in Fig. 
1 1d, our data indicates that the degree of spreading of the distribution (as

2 quantified by the parameter, $\eta^{2}$, Materials and Methods) increases with the

3 surface ratio and decreases when the initial (i.e., apical) Voronoi diagram

4 became more ordered, that is, as the CVT increases (Fig. 2c).

5 Moreover, we computed the average of the total number of contacts of the

6 cells, $\left\langle n_{3 D}\right\rangle$, as a function of the surface ratio and the initial Voronoi diagram

7 (Fig. 2d). Our data are quantitatively consistent with a mathematical derivation

8 that shows that $\left\langle n_{3 D}\right\rangle$ is linearly proportional to the amount of apico-basal

9 intercalations (Materials and Methods and Fig. S1). Also, these results

10 indicated that the average cellular connectivity grows as the tissue thickness

11 and the randomness of cellular organization increases.

12 In summary, our computational model suggests a relation between the 13 tissue geometry (i.e., cell thickness/curvature), the cellular planar topological

14 order (CVT index), and the 3D cellular connectivity in epithelial tubes.

The 3D neighbor's accumulation follows a "poor get richer" principle

17 In order to shed light on the underlying mechanisms that determine the

18 degree of 3D cellular connectivity in tubular epithelia, we computed the net

19 gain of cellular neighbors as a function of the radial expansion

20 (thickness/curvature of tubes) and the topological properties of cells (CVT

21 index and polygonal class at the apical surface). As a general trend, we

22 observed that, independently of the radial expansion, the smaller the number

23 of neighbors at the apical surface the larger the net gain of $3 D$ cellular

24 contacts (Fig. 3a-b, Fig. S2). Additionally, we also checked that this tendency

25 is satisfied when estimating the net gain of neighbors accumulated from the

26 basal to the apical surface (Fig. S2). These results suggest that in tubular

27 epithelia the 3D cell packing follows a "poor get richer" principle driven by

28 apico-basal intercalations: the less neighbors a cell has in a surface (apical or

29 basal), the larger the net increase of cellular contacts. Interestingly, this result

30 is akin to the behavior found in planar geometries that indicates that the

31 probability of undergoing a $\mathrm{T} 1$ transition increases as the number of neighbors

32 decreases (Bi et al, 2014), see Discussion. 
2 An energetics model suggests that cellular connectivity satisfies a 3 logistic-like law

4 In light of this evidence, and in order to better understand the dependence

5 of the tissue self-organization on the radial expansion, $s=R / R_{a}$, we

6 developed a biophysical model (a Kolmogorov rate equation) that accounts for

7 the probability of cells to increase their 3D connectivity driven by energetic

8 cues (Fig. 4a-b and Materials and Methods):

$9 \quad \frac{d P_{m}(s)}{d s}=P_{m-1}(s) r_{m-1, m}-P_{m}(s) r_{m, m+1}$

10 where, $P_{m}$, is the probability of having $m$ accumulated 3D neighbors (i.e.,

$11 m=n_{3 D}$ ) as the surface ratio (i.e., tissue thickness) changes from $s$ to $s+d s$,

12 and $r_{i, i+1}$ accounts for the rate per unit of surface ratio of undergoing an apico-

13 basal intercalation. By drawing parallels between apico-basal intercalations

14 and planar T1 transitions (Sanchez-Corrales et al, 2018; Gómez-Gálvez et al,

15 2018) we assumed that cells need to overcome an energy barrier to gain a 3D

16 neighbor, that is, $r_{i, i+1} \sim e^{-\Delta E_{i}}$ (Fig. 4a-b). The "poor get richer" principle

17 suggests that $\Delta E_{i}$ grows as $i$ increases. In addition, our mathematical

18 calculations proved that the apico-basal intercalation rate becomes null for a

19 finite value of $i$ (Box and Materials and Methods): neighbors' gaining is

20 necessarily bounded or, energetically speaking, the energy barrier to undergo

21 an apico-basal transition becomes eventually infinite. All these facts led to the

22 following expression for the apico-basal intercalation rate: $r_{i, i+1}=\alpha\left(N_{\max }-\right.$

23 i) $e^{-i \beta}$, where $\alpha$ is a 'bare' transition rate, $\beta$ is the dimensionless energy (in

24 units of the four-fold vertex energy configuration) per 3D neighbor that a cell

25 needs to increase its connectivity to an additional cell, and $N_{\max }$ is the

26 maximum 3D cellular connectivity (Materials and Methods).

27 The fitting of the in silico data about the average tissue connectivity, $28\left\langle n_{3 D}(s)\right\rangle=\sum_{m} m P_{m}(s)$, to this biophysical model showed an excellent

29 agreement and confirmed that $\left\langle n_{3 D}\right\rangle>6$ as long as the tissue is subjected to

30 some level of anisotropic curvature (Fig. 4c, Fig. S3 and Materials and

31 Methods). We also observed that the energy required per 3D neighbor to

32 undergo an intercalation, $\beta$, quickly reached a plateau, $\beta \simeq 5 \cdot 10^{-2}$, as the 
1 tissue became more ordered (i.e., as the CVT index increases). Our results

2 also indicate that in Voronoi tubes the scutoidal geometry enables a

3 theoretically increase of the average 3D cellular connectivity up to

$4\left\langle N_{\max }\right\rangle \sim 12-15$ cells (Table S1). In addition, the plausibility of the Kolmogorov

5 approach was further assessed by predicting the 3D neighbor distribution,

$6 \quad P_{m}(s)$, thus confirming that a link between geometrical and energetic traits

7 determines the cellular connectivity in tubular epithelia (Fig. 4c).

8 We also obtained theoretically an analytical formula that characterizes the

9 average 3D cellular connectivity, $\left\langle n_{3 D}\right\rangle$ (Box and Materials and Methods).

10 We concluded that in tubular epithelia $\left\langle n_{3 D}\right\rangle$ can be described by a logistic-like

11 behavior,

$$
\left\langle n_{3 D}(s)\right\rangle \approx\left\langle N_{\max }\right\rangle \frac{1+b e^{-\frac{s}{c}}}{1+d e^{-\frac{s}{c}}}
$$

13 where $b, c$, and $d$ are non-independent parameters that are functions of $\alpha, \beta$,

14 and $\left\langle N_{\max }\right\rangle$ (Table S1 and Materials and Methods). We refer to this logistic-

15 like principle as the "Flintstones' law" after the cartoon characters.

16 The analysis of computational tubes revealed the validity of the Flintstones'

17 law as an effective way to determine the cellular connectivity in tubular

18 epithelia as a function of the radial expansion (Fig. 4c and Fig. S3). More

19 importantly, it provides a straightforward way to estimate/predict the value of

20 the underlying energetic properties regulating apico-basal intercalations and

21 the limiting average 3D cellular connectivity (Table S1).

23 Experiments confirm that the 3D cellular connectivity in tubular epithelia

24 satisfies the Flintstones' law

25 In order to confirm our computational and theoretical predictions, we 26 implemented a novel methodological pipeline that combines several 27 computational image analysis techniques to accurately segment cells of in vivo 28 epithelial tubes (Arganda-Carreras et al, 2017; Machado et al, 2019)

29 (Materials and Methods). We used the Drosophila larval salivary gland as a 30 model due to its ideal characteristics to study complex tubular architectures

31 (Girdler \& Röper, 2014) (Fig. 5a-c). 
1 Our methodology allowed to determine the average surface ratio of the 2 salivary glands $\left(s_{b}=4.0 \pm 0.4\right),\left\langle n_{3 D}\left(s_{b}\right)\right\rangle=6.7 \pm 0.2$, the average percentage

3 of scutoids $(76 \pm 11 \%)$, and the average number of apico-basal intercalations

4 per cell, $\left\langle i\left(s_{b}\right)\right\rangle=1.4 \pm 0.4$, thus confirming the validity of the formula that

5 relates apico-basal intercalations per cell and the average connectivity:

$6\left\langle n_{3 D}\right\rangle=6+\langle i\rangle / 2$ (Materials and Methods and Fig. S1). We also calculated

7 the polygonal class distribution in the apical and basal surfaces (Fig. S4).

8 Interestingly, in spite of the prevalence of scutoids, the polygonal organization

9 of apical and basal surfaces was found to be the same and equivalent to that

10 obtained in in silico V8 tubes with a radial expansion $s_{b}=1.75$ (Fig. S4). This

11 V8 model $\left(s_{b}=1.75\right)$ also displayed a similar scutoidal prevalence (79 $\left.\pm 5 \%\right)$,

12 average number of 3D neighbors, average number of apico-basal

13 intercalations per cell, and $\eta^{2}$ spreading that in vivo tubes (Fig. 5d and Fig.

14 S4). We additionally confirmed that the apical and basal surfaces of the V8

15 model and the salivary glands fulfilled, as expected, that $\left\langle n_{2 D}\right\rangle \approx 6$ (Reinhardt,

16 1918; Wetzel, 1926) (Fig. S4). Thus, we concluded that the in silico V8 model

17 with a radial expansion of $s_{b}=1.75$ faithfully recapitulates the $3 \mathrm{D}$ packing

18 properties of in vivo salivary glands.

19 As for the 3D cellular connectivity of in vivo tubes, our analyses confirmed 20 that the "poor get richer" principle was satisfied, thus supporting the idea that 21 the smaller the number of neighbors of a cell in a surface, the larger the 22 probability to increase its connectivity (Fig. S4). Additionally, by implementing 23 an un-rolling (i.e., peel-off) algorithm (Yang et al, 2019) (Materials and

24 Methods), we obtained concentric radial sections and quantified the number 25 of $3 \mathrm{D}$ neighbors as a function of the radial expansion. The fitting of the data to 26 the Kolmogorov model showed an excellent agreement and allowed to 27 estimate the energetic properties as summarized by the parameter $\beta$ (Fig. 5e 28 and Table S1). Our results suggested that the energy per 3D cell required to 29 undergo an apico-basal intercalation is larger in in vivo tubes than in the 30 computational V8 model, see Discussion. Importantly, the 3D cellular 31 connectivity data confirmed the applicability of the Flintstones' law in in vivo 32 tubular epithelia (Fig. 5e and Table S1). 


\section{DISCUSSION}

2 Here we have shown how mathematical and physical principles underlie the

3 emergence of functionally complex 3D developmental structures, e.g., glands.

4 Our analyses have uncovered for the first time how a 2D organizational trait,

5 i.e., the cellular connectivity, can be extended to the third spatial dimension

6 when the novel paradigm of epithelial cells' shapes and packing, the scutoid,

7 is considered. In that regard, we have revealed how the 3D cellular

8 connectivity and tissue energetics are coupled, and we have stated a novel

9 principle, the so-called Flintstones' law. The latter links the activation energy

10 needed to recruit additional neighbors with geometrical descriptors (i.e., tissue

11 thickness/curvature). Our results provide new biological insight into the

12 spatiotemporal regulation of cell-cell connectivity, a property that ultimately

13 regulates juxtracrine signaling and is pivotal for primordia patterning and cell

14 fate determination (Tung et al, 2012; Sharma et al, 2019). In this context, our

15 study points towards an effect of scutoids on the regulation of the physiological

16 properties of tissues. Therefore, our findings, on top of being fundamental to

17 understand self-organization of epithelia in 3D, open new ways to investigate,

18 and draw implications about, primary developmental processes in which

19 epithelial bending is essential such as tubulogenesis, gastrulation, or

20 neurulation.

21 Our study also provides important methodological advances. Previous

22 software developed to identify the outlines of the epithelial cells does not work

23 on $3 \mathrm{D}$ or lacks enough precision to extract the geometrical and topological

24 data needed to quantify tissue packing in 3D (Khan et al, 2014; Heller et al,

25 2016; Bassel et al, 2014; Gómez-Gálvez et al, 2018). Here we have shown

26 that our methodological pipeline (Materials and Methods) allows to

27 implement a 3D segmentation and the precise reconstruction of cells in

28 epithelia subjected to curvature. We stress that this level of detail is necessary

29 to be able to quantify the apico-basal intercalation phenomenon and,

30 therefore, compare the results with the computational models and extract

31 biological consequences. We then argue that our methodology, by enabling

32 the analysis of $3 \mathrm{D}$ packing in a realistic way, will benefit the field of

33 morphogenesis by bringing understanding about the cellular and mechanical 
1 basis of self-organization in curved tissues (Inoue et al, 2019; Ambrosini et al,

2 2017; Hirashima \& Adachi, 2019) or even whole embryos (Shahbazi et al,

3 2019). In addition, our analysis indicates that the Flintstones' law can be used

4 quantitatively to estimate key connectivity-related parameters, e.g., $\beta$ and/or

$5\left\langle N_{\max }\right\rangle$. This avoids the burden of solving the optimization problem associated

6 with the Kolmogorov model that is computationally demanding (Material and

7 Methods). Thus, the values obtained by means of fittings to the Flintstones'

8 law are, at the very least, within the same order of magnitude with respect to

9 the 'exact' Kolmogorov calculations (Table S1). This reveals the usability of

10 the Flintstones' law not just as a principle that is satisfied by tubular epithelia,

11 but as a practical way to connect packing properties, geometrical descriptors,

12 and biophysical traits due to its predictive character.

13 As a matter of discussion, the connectivity law that we have introduced 14 herein, depends on a prediction obtained from the Voronoi computational 15 model that was confirmed in experiments: the "poor get richer" principle. 16 Roughly speaking, we have shown that the fewer neighbors a cell has on a

17 surface, the larger is the probability of a connectivity increase. Interestingly, a 18 similar idea has been reported in $\mathrm{T} 1$ dynamical processes during the 19 remodeling of planar epithelia (Bi et al, 2014). Since the scutoidal geometry 20 can be related to planar T1 transitions by exchanging the concepts of space 21 and time, this result reinforces the idea of the existence of universal principles 22 driving the organization of tissues.

23 In our study we have found that in real tissues the energy cost per 3D 24 neighbor that a cell requires to increase its connectivity, $\beta$, is larger than in 25 Voronoi models. We hypothesize that it is due to the purely geometrical 26 description used in the latter. That is, while in in silico models the apico-basal 27 transitions develop just a result of a topological constraint (Voronoi 28 tessellation), in the salivary glands, on top of geometrical requirements, the 29 cells must actively remodel their cytoskeleton to make the transitions possible.

30 That component would explain the larger effective cost of gaining new 31 neighbors in real tissues. The reduced energetic cost for gaining neighbors in 32 the Voronoi computational approach also explains why in silico tubes led to a 33 larger limiting average 3D cellular connectivity, $\left\langle N_{\max }\right\rangle$, and the V8 model with 
1 the same surface ratio that the salivary gland, $s_{b}=4$, developed more apico-

2 basal transitions than the real samples (Fig. 5e, Fig. S4, and Table S1).

3 These data ultimately explain why it is necessary to rescale appropriately $s_{b}$ to

4 obtain a computational model with packing, topological, and connectivity

5 properties similar to those of the salivary glands. In that regard, our results

6 suggest that salivary glands are optimized to reach a high cellular connectivity.

7 While the in silico V8 model with a radial expansion of $s_{b}=1.75$ or $s_{b}=4$ are

8 far for reaching their maximum average connectivity (i.e., $\left\langle n_{3 D}\right\rangle$ 's are,

9 respectively, $\sim 47 \%$ and $\sim 61 \%$ of $\left.\left\langle N_{\max }\right\rangle\right)$, in the salivary glands $\left\langle n_{3 D}\left(s_{b}\right)\right\rangle$ is

$10 \sim 87 \%$ of $\left\langle N_{\max }\right\rangle$ (Fig. 5e, Fig. S4, and Table S1). This optimization could be

11 related to a functionality improvement of the gland, similarly to what has been

12 suggested in pituitary growth hormone secretory cells, where the increase of

13 3D cellular connectivity has been proposed to better coordinate the pulses of

14 hormone secretion (Bonnefont et al, 2005).

15 As for the broader implications of our findings, we argue that, while our 16 analyses focus on static tissues from the point of view of tissue architecture,

17 our results can also be relevant to understand active 3D tissue remodeling

18 (e.g., fluidization). Recent studies have revealed that active remodeling

19 involves changes in the material-like properties of tissues that can be

20 connected to an increased activity of neighbor exchanges (Tetley et al, 2019;

21 Mongera et al, 2018). In that regard, here we have shown that the physical

22 basis of 3D self-organization (i.e., 3D cellular packing and connectivity) in

23 tubular epithelia effectively relies on a constant amount of energy, $\beta$. Thus,

24 arguably, active 3D tissue remodeling would imply dynamical changes on the

25 value of $\beta$ that would modify the apico-basal intercalation propensity and

26 therefore the material-like properties: the larger $\beta$ the more solid-like the tissue

27 would behave. Finally, with respect to the applicability of our results to other

28 areas, we expect that the emerging field of organoids will benefit from our

29 discoveries. A precise quantification of 3D connectivity could then help to

30 understand the lack of reproducibility in organoid production, one of the

31 biggest challenges of the field (Schutgens et al, 2019; Huch et al, 2017;

32 Clevers, 2016). Also, from a medical point of view, it has been recently shown

33 that tissue curvature affects tumor progression due to the imbalance of 
1 tensions in apical and basal surfaces of epithelial tubes (Messal et al, 2019).

2 The Flintstones' law explains how energetic cues affect the 3D packing of

3 these cells and therefore may shed light on the mechanism of tumorigenic

4 morphogenesis in tubular organs.

\section{MATERIALS AND METHODS}

\section{Immunohistochemistry and confocal imaging of salivary glands}

$7 \quad$ Flies were grown at $25^{\circ} \mathrm{C}$ using standard culture techniques. We dissected

8 the salivary glands from third instar larvae of the wild type Oregon $R$ strain.

9 After PBS dissection, the glands were fixed using 4\% paraformaldehyde in

10 PBS for $20 \mathrm{~min}$. The samples were washed three times for $10 \mathrm{~min}$ with PBT

11 (PBS, 0.3\% Triton) and then incubated for $1 \mathrm{hr} 45$ minutes at room

12 temperature with Cy3-labeled phalloidin (Sigma) to label the cell contours of

13 the epithelial cells. Stained larval salivary glands were mounted using

14 Fluoromount-G (Southern Biotech). We used two pieces of double-sided

15 adhesive tape (one on top of each other) as a spacer (Aldaz et al, 2013), so

16 the salivary glands preserve their shape. Images were taken using a Nikon

17 Eclipse Ti-E laser scanning confocal microscope. The images were captured

18 using a $\times 20$ dry objective and $2.5 \mu \mathrm{m}$ steps between slices. The image stacks

19 were exported as $1024 \times 1024$ pixels TIFF files.

\section{3D glands segmentation}

21 To segment the salivary gland stacks of images and reconstruct (semi22 automatically) the shape of cells in three dimensions we used the FIJI

23 (Schindelin et al, 2012) plugin LimeSeg (Machado et al, 2019). We inferred

24 cell outlines by using surface elements ("Surfels") obtained by placing single

25 ellipsoidal-like seeds on every cell (see https://imagej.net/LimeSeg for details).

26 Once cell outlines were found (Fig. 5b-c), we exported them as point clouds

27 (output). We developed a custom-made Matlab code (2018a MathWorks) to 28 postprocess the output of LimeSeg in order to correct errors and obtain 29 perfectly segmented salivary glands. In addition, we manually segmented the 30 lumen of the glands from the images using Adobe Photoshop CS6 and 31 reconstructed it using a Matlab code. To faithfully represent the gland as a 32 cylinder, we selected a subset of cells: cells that were not ductal, neither 
1 located at the tip of the gland. For more information about the processing

2 pipeline: https://osf.io/nd5t6/.

3 To obtain the cellular neighborhood relations of salivary glands for different

4 values of the radial expansion, we proceeded as follows. We calculated the

5 cell height by estimating the distance between the average voxel positions of

6 the apical surface with respect to the average voxel positions of its basal

7 surface, $d\left(s_{a}, s_{b}\right)$. Then, to capture a concentric radial section of the gland, we

8 linearly extrapolated the equivalent cell height to the given surface ratio, $s$ :

$$
d\left(s_{a}, s\right)=d\left(s_{a}, s_{b}\right) \frac{s}{s_{b}}
$$

10 where $d\left(s_{a}, s\right)$ is the Euclidean distance between the position of the centroid of

11 the cell in apical and that the position of the centroid at a value $s$ of the radial

12 expansion. Finally, to obtain the gland cylindrical radial section for a given

13 value of the radial expansion, $s$, we collected all voxels between apical and the

14 upper bound of the calculated distance $d\left(s_{a}, s\right)$. Those cylindrical surfaces of

15 the salivary gland were mapped in the Cartesian plane for analysis using a

16 cylindrical coordinates transformation.

\section{Salivary glands measurements}

18 We quantified the following geometrical and topological descriptors of the 19 segmented salivary glands using a custom-made Matlab code:

- Surface ratio expansion (s): Assuming a cylindrical shape for glands, we estimated $s$ by dividing the area of the basal surface of glands by area of the apical surface.

Likewise, we carried out the calculations of the percentage of scutoids and

27 the number of apico-basal transitions.

\section{Voronoi tubular model}

29 Using custom-made Matlab code (R2018a) we generated a Voronoi model 30 that simulates the surface of a cylinder unfolded over the Cartesian plane, see 31 details in Gomez-Galvez et al. ((Gómez-Gálvez et al, 2018), Material and 
1 Methods). The only difference with the cited methodology, is that in this work

2 the Voronoi diagrams has been constructed by means of the Delaunay

3 triangulation technique. Therefore, we just considered the cells' vertices

4 information (cartesian coordinates and connections) for a much faster

5 computation. For each realization, we used an initial set of 200 randomly

6 located seeds on a rectangular domain of 512 ( $X$ axis; transverse axis of

7 cylinder) per 4096 ( $Y$ axis; longitudinal axis of cylinder). In total, we

8 implemented 20 different realizations (i.e., tubes). We performed this

9 procedure for 10 different initial Voronoi diagrams (Voronoi 1 (V1, random

10 seeds) to Voronoi 10 (V10, more ordered and homogeneous cells). These

11 diagrams represent the apical (inner) surfaces of computational tubes, and

12 they were obtained by applying N-1 times the Lloyd's algorithm (Lloyd, 1982)

13 to the random seeds, where $\mathrm{N}$ is then the resulting Voronoi model. For

14 instance, to compute a V1, we use purely random seeds, while to obtain a V4

15 diagram, it would be required to apply 3 times the Lloyd's algorithm to random

16 seeds. V8 diagrams provide a polygonal organization in apical surfaces as

17 experimentally observed (Main Text and Fig. S4). Subsequent radial sections

18 that define computational tubes with different surface ratios were obtained by

19 implementing a radial projection of the Voronoi seeds. For each apical surface

20 of the tube, we generated 40 expansions by incrementing the surface ratios

$21\left(s_{b}\right)$ using 0.25 steps: 1 (apical), 1.25, 1.5, ..,10 (maximum basal surface).

22 As for the 3D reconstruction of cells in Voronoi tubes, each set of seeds that

23 characterizes cells on a given cylindrical section defines a unique 2D Voronoi

24 diagram at every surface and hence the corresponding 2D cellular domains.

25 The set of 2D Voronoi regions that belong to the same radially projected seed

26 from the apical to the basal surface then define each 3D cellular shape. Each

27 of the obtained 3D Voronoi cells was further processed using the Matlab

28 function 'alphaShape' to transform the set of voxels into a compact, solid,

29 object. This reconstruction pipeline was implemented using Matlab (2018a).

30 Code available at https://osf.io/nd5t6/.

31 Voronoi tubular model measurements.

32 We measured the following properties of cells in Voronoi tubular models:

33 number of sides of cells for a given radial section, and total number neighbors. 
1 Additionally, we computed the percentage of scutoids, the number of apico-

2 basal transitions, the polygon distribution of every surface (radial sections). In

3 these quantifications, we disregarded cells at the boundaries (tips of tubes) to

4 avoid 'border effects'.

\section{$5 \quad$ In Voronoi tubes the net gain of 3D neighbors is bounded}

6 Assuming a cylindrical geometry (e.g., epithelial tubes), each point at a given

7 radial surface can be represented into the Cartesian plane; where coordinate $x$

8 accounts for the cylindrical transversal coordinate and coordinate $y$ for the

9 longitudinal one (see Box). Thus, if the coordinates of a point (e.g., a Voronoi

10 seed) at the apical surface are given by $(x, y)$, the coordinates of that point at

11 a surface with a value of the cylindrical radial expansion $s \in[1, \infty)$ can be

12 found by defining the function $f_{s}: \mathbb{R}^{2} \rightarrow \mathbb{R}^{2} f_{s}(x, y)=(s x, y)$. Under these

13 conditions, we aim to characterize the seeds that generate scutoids

14 (exchanges in the neighboring relations of seeds) as $s$ changes.

15 Lemma 1. Given three non-colinear points $\{A, B, C\}$ that define a circle (a 16 nearest-neighbors relation), and another exterior point $D$, if $s>1$ exists such 17 that $f_{s}(D)$ is interior to the circle defined by $\left\{f_{s}(A), f_{S}(B), f_{s}(C)\right\}$, then $D$ is inside 18 of the vertical parabola containing $\{A, B, C\}$ (Box).

19 Remark. If two of the three points $\{A, B, C\}$ are on the same vertical line, then 20 the parabola considered in Lemma 1 degenerates as a vertical strip. Even in 21 this case, the thesis of the Lemma is true if we replace the interior of the 22 parabola by the inside of the strip.

23 Proof. Without loss of generality, we can suppose that $\{A, B, C\}$ are 24 counterclockwise oriented. Thus, the point $D(x, y)$ is outside the circle defined 25 by $\{A, B, C\}$ if, and only if, the sign of the following determinant is negative:

$$
\left|\begin{array}{llll}
a_{1} & a_{2} & a_{1}^{2}+a_{2}^{2} & 1 \\
b_{1} & b_{2} & b_{1}^{2}+b_{2}^{2} & 1 \\
c_{1} & c_{2} & c_{1}^{2}+c_{2}^{2} & 1 \\
x & y & x^{2}+y^{2} & 1
\end{array}\right|=\left|\begin{array}{cccc}
a_{1} & a_{2} & a_{1}^{2} & 1 \\
b_{1} & b_{2} & b_{1}^{2} & 1 \\
c_{1} & c_{2} & c_{1}^{2} & 1 \\
x & y & x^{2} & 1
\end{array}\right|+\left|\begin{array}{llll}
a_{1} & a_{2} & a_{2}^{2} & 1 \\
b_{1} & b_{2} & b_{2}^{2} & 1 \\
c_{1} & c_{2} & c_{2}^{2} & 1 \\
x & y & y^{2} & 1
\end{array}\right|<0
$$

27 For the sake of simplicity, we represent the previous equation as:

$$
\operatorname{det}(\mathcal{A})=\operatorname{det}(\mathcal{B})+\operatorname{det}(\mathcal{C})<0
$$


1 On the other hand, by considering $x$ and $y$ as variables, the equation

$2 \operatorname{det}(\mathcal{A})=0$ corresponds to the circle defined by $\{A, B, C\}$, and $\operatorname{det}(\mathcal{B})=0$

3 corresponds to the vertical parabola defined by the same three points.

4 Consequently, the inequality $\operatorname{det}(\mathcal{B})>0$ defines the locus of interior points to

5 that parabola.

6 Now, assuming that $s>1$ exists such that $f_{s}(D)$ is interior to the circle

7 defined by $\left\{f_{s}(A), f_{s}(B), f_{s}(C)\right\}$. Then,

$$
\left|\begin{array}{cccc}
s a_{1} & a_{2} & s^{2} a_{1}^{2}+a_{2}^{2} & 1 \\
s b_{1} & b_{2} & s^{2} b_{1}^{2}+b_{2}^{2} & 1 \\
s c_{1} & c_{2} & s^{2} c_{1}^{2}+c_{2}^{2} & 1 \\
s x & y & s^{2} x^{2}+y^{2} & 1
\end{array}\right|=s^{3} \operatorname{det}(\mathcal{B})+s \operatorname{det}(\mathcal{C})>0
$$

8 Or, equivalently, $s^{2} \operatorname{det}(\mathcal{B})+\operatorname{det}(\mathcal{C})>0, \quad$ so, $\quad s^{2} \operatorname{det}(\mathcal{B})>-\operatorname{det}(\mathcal{C})$. If

$9 \operatorname{det}(\mathcal{B})<0$, then $1<s^{2}<-\frac{\operatorname{det}(\mathcal{C})}{\operatorname{det}(\mathcal{B})}$ and therefore $\operatorname{det}(\mathcal{B})>-\operatorname{det}(\mathcal{C})$. The latter

10 is in contradiction with $\operatorname{det}(\mathcal{B})+\operatorname{det}(\mathcal{C})<0$. As a result, $\operatorname{det}(\mathcal{B})>0$, and the

11 following inequality holds,

$$
s^{2}>-\frac{\operatorname{det}(\mathcal{C})}{\operatorname{det}(\mathcal{B})}>1
$$

12 Notice that if the circle defined by $\{A, B, C\}$ is surrounded by a set of points 13 and we change continuously the parameter $s$ in the interval $[1, \infty)$, it is 14 possible to detect the first point touching the circle defined by $15\left\{f_{s}(A), f_{S}(B), f_{S}(C)\right\}$. That point can be obtained by computing all the points at

$16 s=\sqrt{-\frac{\operatorname{det}(\mathcal{C})}{\operatorname{det}(\mathcal{B})}}$. Hence, the first point contacting the circle will be that with the 17 minimum value of $s$.

18 As for proving that the average of the number of neighbours of a cell induced 19 by a seed grows is bounded as a function of the surface ratio, we state the 20 following proposition:

21 Proposition 1. Given a Voronoi seed representing a cell, if $n_{3 D}(s)$ is the total 22 number of accumulated cell neighbors as $s$ increases from $s=1$ (apical 23 surface) to a given value of $s$, then $\left\langle n_{3 D}(s)\right\rangle$ is a bounded function for a finite 24 cylinder. 
1 Proof. We model the apical surface as the cylinder $2 \pi r \times h$, where $r$ 2 representes the inner radius and $h$ the length of the cylinder. Given a seed $A$

3 in that surface, in the corresponding Delaunay triangulation it appears as a

4 point surrounded by triangles defining the neighbourhood of $A$. By Lemma 1 ,

5 each triangle defines a vertical parabola and a circle. So, any other seed

6 touching $A$ in other layer must be inside of one of the parabolas and outside of

7 all circles (see Box). Let's denote $\mathcal{R}_{S, A}$ the feasible region for a new neighbour

8 of $A$ in the layer represented by $s$, i.e., all points inside one of the parabolas

9 and outside all the circles. Thus, if $\#\left(\mathcal{R}_{s, A}\right)$ is the number of seeds in that

10 region that are not neighbours of $A$ in the apical surface, obviously, an upper

11 bound to the number of new neighbours to $A$ is given by $\#\left(\mathcal{R}_{s, A}\right) \leq \#\left(\mathcal{R}_{1, A}\right)$.

13 On the other hand, that number of seeds is, in average, proportional to the 14 density of seeds times the area of $\mathcal{R}_{s, A}$, therefore, the average number of

15 accumulated neighbours of $A$, denoted as $\left\langle n_{3 D}(A)\right\rangle$, will be bounded by the

16 change of the density of points when growing $s$, this is to say,

$$
d\left\langle n_{3 D}(A)\right\rangle \leq M \cdot \frac{\mathcal{R}_{S, A}}{2 \pi s r \cdot h} d s
$$

where $M$ represents the total number of seeds (i.e., the total number of cells that is a constant) and the quotient is the area of $\mathcal{R}_{s, A}$ divided by the area of a given radial layer. In general, it is not possible to integrate equation (4), since the area of $\mathcal{R}_{S, A}$ is known only in very few, particular, cases.

If the case of a finite cylinder, $\left\langle n_{3 D}(A)\right\rangle \leq \#\left(\mathcal{R}_{S, A}\right) \leq \#\left(\mathcal{R}_{1, A}\right)$ leads, suming up to all the seeds and dividing by $M$, to the upper bound

$$
\left\langle n_{3 D}(s)\right\rangle \leq \frac{1}{M} \cdot \sum_{A} \#\left(\mathcal{R}_{1, A}\right)
$$

30 thus, $\left\langle n_{3 D}(s)\right\rangle$ is necessarily a bounded function. This expression indicates

31 that the number of new neighbours when increasing $s$ exhausts since the

32 number of cells is a resource shared by all the layers. It is possible to obtain 
1 an upper bound to $\left\langle N_{\max }\right\rangle=\lim _{s \rightarrow \infty}\left\langle n_{3 D}(s)\right\rangle$ since, after a flip in the Delaunay

2 triangulation, the edge disappearing (i.e., a cell contact loss) can never be

3 recovered in a cylindrical geometry. Thus, $M \cdot\left(\left\langle N_{\max }\right\rangle-n_{3 D}(1)\right)$ is bounded

4 by the number of edges that complement the original Delaunay triangulation

5 on the apical surface, that is,

6

$$
\left\langle N_{\max }\right\rangle-\left\langle n_{3 D}(1)\right\rangle \leq \frac{1}{M} \cdot\left(\frac{M(M-1)}{2}-M \frac{\left\langle n_{3 D}(1)\right\rangle}{2}\right)=\frac{M-1}{2}-\frac{\left\langle n_{3 D}(1)\right\rangle}{2}(10)
$$

leading to

$$
\left\langle N_{\max }\right\rangle \leq \frac{M-1}{2}+\frac{\left\langle n_{3 D}(1)\right\rangle}{2} \leq \frac{M-1}{2}+3=\frac{M+5}{2}(11)
$$

Where we have assumed that $\left\langle n_{3 D}(1)\right\rangle=6$. The simulations of the computational Voronoi model and the data of the salivary gland show that $\left\langle N_{\max }\right\rangle$ is in fact much smaller that the theoretical bound $\frac{M+5}{2}$.

\section{Relation between total accumulated 3D neighbors and the number of intercalation events}

Scutoids have a Euler characteristic $\chi=2$ such that $V-E+F=2$, where $V$, $E$, and $F$ accounts for the number of vertexes, edges, and faces respectively. We assumed that the apical, $a$, and basal, $b$, faces of scutoids tesellating a cylindrical space have radial coordinates $R_{a}$ and $R_{b}$ respectively. Then, for any value of the surface ratio expansion, $s=R / R_{a}$, these solids can be mapped into a connected plane graph with the same Euler characteristic (a sort of projection of the vertexes and connectors into the plane, see Fig. S5. Thus, as a function of $s$, the accumulated number of 3D neighbors reads $n_{3 D}(s)=$ $E(s)-V(s)$. Since in tubular geometries the centroids of tessellating scutoids always separate from each other as $s$ increases (i.e., apico-basal intercalations are not reversible) for a single scutoid,

$$
n_{3 D}(s)=\max (\{V(s)\})=\min (\{V(s)\})+i(s)
$$

where $\{V(s)\}=\left\{V(1), V(1+d s), \cdots, V\left(s_{b}\right)\right\}$ and $i(s)$ denotes the number of intercalation points in the interval $s \in\left[1, s_{b}\right]$. In the case of a 3D tessellation with $N$ cells, where $M$ of them do not show any intercalation, the total number of accumulated neighbors reads, 
Given that each intercalation point is shared by four cells, two of them necessarily increase their number of vertices in a given $s$-plane and two of them decrease their number of vertices (see Fig. 1a). Thus, in the case of a decrease $\max \left(\left\{V^{(j)}(s)\right\}\right)=V^{(j)}(1)$ and in the case of an increase $\min \left(\left\{V^{(j)}(s)\right\}\right)+i^{(j)}(s)=V^{(j)}(1)+i^{(j)}(s)$. Consequently,

$$
n_{3 D}(s)=\sum_{j=1}^{N} V^{(j)}(1)+\sum_{j=1}^{(N-M) / 2} i^{(j)}(s)=\sum_{j=1}^{N} V^{(j)}(1)+\frac{1}{2} \sum_{j=1}^{N-M} i^{(j)}(s)
$$

where we used the fact that for every intercalation event that increases by one the number of neighbors there is one that decreases the number of neighbors in the same amount; consequently, we can add up all intercalation events and divide by two. Hence the average number of accumulated 3D neighbors, $\left\langle n_{3 D}(s)\right\rangle=n_{3 D}(s) / N$ reads $\left\langle n_{3 D}(s)\right\rangle=\langle V(1)\rangle+\langle i(s)\rangle / 2 ;\langle i(s)\rangle$ being the average number of apico-basal intercalations per cell. Finally, by considering that any $s$-surface, and in particular the apical surface $s=1$, corresponds to a $2 \mathrm{D}$ tessellation of convex polygons, $\langle V(1)\rangle=6$ we conclude that,

$$
\left\langle n_{3 D}(s)\right\rangle=6+\frac{1}{2}\langle i(s)\rangle
$$

\section{A Kolmogorov rate equation for the 3D cellular connectivity}

The probability, $P$, of having $m$ accumulated 3D neighbors (i.e., $m=n_{3 D}$ ) as the surface ratio increases from $s$ to $s+d s$ can be described by the following Markov equation (Fig. 4b),

$$
P_{m}(s+d s)=P_{m}(s) T_{m, m}+P_{m-1}(s) T_{m-1, m}
$$

where $T_{i, j}$ is the probability of incrementing the number of neighbors from $i$ to $j$ due to an apico-basal intercalation. Since $\sum_{j} T_{i, j}=1$ (normalization of the transition probabilities) and $T_{i, j}=f(i, j)\left\{\delta_{i-1, j}+\delta_{i, j+1}\right\}$ (each intercalation can only possibly induce to win one neighbor) then $T_{m, m}=1-T_{m, m+1}$ and the 
1 above Markov equation can be written as a Kolmogorov equation (a.k.a.

2 Master equation):

$$
\frac{d P_{m}(s)}{d s}=P_{m-1}(s) r_{m-1, m}-P_{m}(s) r_{m, m+1}
$$

4 where $r_{i, j}$ accounts for the probability of apico-basal intercalations per unit of

5 surface ratio, i.e., $T_{i, j}=r_{i, j} d s$.

6 If we assume an Arrhenius-like kinetics ( $\mathrm{Bi}$ et al, 2014) then $r_{i, i+1}=\hat{\alpha} e^{-\Delta E_{i}}$,

7 where $\hat{\alpha}$ is the so-called pre-exponential factor that modulates the "bare"

8 frequency of intercalations (per unit of surface ratio expansion) and $\Delta E_{i}$ is the

9 activation energy in some energy units, e.g., in units of $E_{0}$ (the value of the

10 energetic barrier of the four-fold vertex configuration). The observed "poor get

11 richer" behavior suggests that the activation energy, $\Delta E_{i}$, increases with $i$. For

12 the sake of simplicity, up to first order in $i: \Delta E_{i}=i \cdot \beta$ ( $\beta$ being the

13 dimensionless activation energy of a cell per 3D neighbor in units of $E_{0}$ ). On

14 the other hand, the mathematical calculations (see Eq. (9)) indicate that the

15 intercalation rate $r_{i, i+1}$ becomes null for a finite value of $i$ or, alternatively, that

16 the activation energy becomes infinite for a finite value of $i$. Otherwise, the net

17 gain of new neighbors is not bounded. This fact can be accounted for by

18 assuming that the bare frequency is a function of the number of neighbors,

$19 \hat{\alpha}=\hat{\alpha}(i)$, such that $\frac{d \hat{\alpha}}{d i}<0$ and becomes null for a finite value of $i$. Again, for

20 the sake of simplicity, we assume that up to first order in $i: \hat{\alpha}=\alpha\left(N_{\max }-i\right)$,

21 where $N_{\max }$ is the asymptotic, maximum, number of 3D neighbors a cell can

22 possibly have. Summarizing, we assume that the apico-basal intercalation rate

$23 r_{i, i+1}$ reads,

$24 \quad r_{i, i+1}=\alpha\left(N_{\max }-i\right) e^{-i \beta}(18)$

25 Under these conditions, the Kolmogorov equation reads,

$$
\frac{d P_{m}(s)}{d s}=\alpha\left(N_{\max }-(m-1)\right) e^{-\beta(m-1)} P_{m-1}(s)-\alpha\left(N_{\max }-m\right) e^{-\beta m} P_{m}(s)
$$

28 On the other hand, the equation satisfied by the average number of 29 accumulated 3D neighbors, $\left\langle n_{3 D}\right\rangle=\langle m\rangle$, reads, 


$$
\frac{d\langle m(s)\rangle}{d s}=\sum_{m} m \frac{d P_{m}(s)}{d s}=\sum_{m} r_{m, m+1} P_{m}(s)=\left\langle r_{m, m+1}\right\rangle
$$

2 Alternatively, in order to obtain an analytical expression able to recapitulate,

3 effectively, the mathematical principle that govern the net gain of 3D

4 neighbors, we perform the following approximations. First, we perform a

5 mean-field-like approximation, i.e., $\langle F(m)\rangle \approx F(\langle m\rangle)$,

$$
\frac{d\langle m\rangle}{d s} \approx \alpha\left(\left\langle N_{\max }\right\rangle-\langle m\rangle\right) e^{-\beta\langle m\rangle}
$$

7 Where $\left\langle N_{\max }\right\rangle$ is the limiting average cellular connectivity. Second, since $\beta<$ 81 ,

$$
\frac{d\langle m\rangle}{d s} \approx \alpha\left(\left\langle N_{\max }\right\rangle-\langle m\rangle\right)(1-\beta\langle m\rangle)+\mathcal{O}\left(\beta^{2}\right)
$$

10 Equation (21) is formally a logistic-like growth equation,

$$
\frac{d\langle m\rangle}{d s}=\frac{b}{c(b-d)}\left(\left\langle N_{\max }\right\rangle-\langle m\rangle\right)\left(1-\frac{d}{b N_{\max }}\langle m\rangle\right)
$$

12 that has as solution,

$$
\langle m(s)\rangle=\left\langle N_{\max }\right\rangle \frac{1+b e^{-\frac{s}{c}}}{1+d e^{-\frac{s}{c}}}
$$

14 Thus, if $c>0$ then $\lim _{s \rightarrow \infty}\langle m(s)\rangle=\left\langle N_{\text {max }}\right\rangle$. The parameters $b, c$, and $d$ are

15 further constrained by the following facts: $\frac{d\langle m\rangle}{d s}>0$ (3D neighbors can only 16 accumulate) and $\frac{d^{2}\langle m\rangle}{d s^{2}}<0$ ("poor get richer" principle). Moreover, if we impose

17 the condition $\langle m(1)\rangle=6$ (the average number of neighbors in the apical 18 surface is 6) these parameters are not independent since,

$$
b=\frac{6 d-\left(\left\langle N_{\max }\right\rangle-6\right) e^{\frac{1}{c}}}{\left\langle N_{\max }\right\rangle}
$$

20 All the above implies that the logistic-like fitting function, Eq. (24), describes, 21 approximately but effectively, the analytical mathematical law ("Flintstone's 22 law") underlying the 3D average connectivity if the following conditions hold, 23 either $-1<d<0$ or $c \ln (-d)<1$ if $d<-1$.

24 The relation between the fitting parameters of the logistic fitting with $\alpha$ and $\beta$ 25 are, 


$$
\begin{aligned}
& \alpha=\frac{b}{c(b-d)} \\
& \beta=\frac{d}{b\left\langle N_{\max }\right\rangle}
\end{aligned}
$$

3 For finding the parameters $\alpha$ and $\beta$ in in silico tubes and salivary glands we

4 then implemented two possible approaches. On the one hand, we

5 implemented an error minimization algorithm that recursively solved,

6 numerically, Eq. (20) to obtain $\langle m(s)\rangle=\sum_{m} m P_{m}(s)$ taking also into account

7 the normalization condition $\sum_{m=1}^{\infty} P_{m}(s)=1$ (code available at

8 https://osf.io/nd5t6).

9 On the other hand, we obtained values using the fitting logistic function Eq.

10 (24). We notice that the values obtained through the first method are exact as

11 compared to the values obtained from the fitting that are based on a series of

12 approximations as explained above (see Table S1).

13 The values of $\alpha$ and $\beta$ are obtained from the exact methodology were further

14 used to compare the predicted probability distribution of having $m$

15 accumulated neighbors for a given value of $s: P_{m}(s)$. We evaluated the relative

16 error of this prediction with respect to the actual distribution from data,

$17 P_{m}^{a c t u a l}(s)$, by computing $\varepsilon^{2}=\frac{1}{2} \sum_{m}\left(P_{m}^{a c t u a l}(s)-P_{m}(s)\right)^{2}$. This quantity is

18 normalized such that in case of the following situation of full disagreement

19 between the distributions, $P_{m}^{\text {actual }}(s)=\delta_{m, i}$ and $P_{m}(s)=\delta_{m, j}$ with $i \neq j$,

20 provides $\varepsilon^{2}=1$ (i.e., $100 \%$ error).

22 Quantitative characterization of spreading in neighbor exchange 23 distributions between apical and basal surfaces

24 In order to characterize the spreading away from the diagonal in neighbor 25 exchange distributions between apical and basal surfaces, Fig. 2a-b, we 26 followed the same approach used to quantify intrinsic noise during gene

27 expression processes (see (Elowitz, 2002). Thus, $\eta^{2}=\frac{\left\langle\left(n_{a}-n_{b}\right)^{2}\right\rangle}{2\left\langle n_{a}\right\rangle\left\langle n_{b}\right\rangle}$ where $28\left\langle z\left(n_{a}, n_{b}\right)\right\rangle=\sum_{n_{a}, n_{b}} z\left(n_{a}, n_{b}\right) p\left(n_{a}, n_{b}\right) ; z$ representing any function of $n_{a}$ and $n_{b}$ 29 and $p\left(n_{a}, n_{b}\right)$ being the probability of neighbor exchange events.

\section{$30 \quad$ Logistic data fitting}


1 To obtain the logistic function that fit best our data points, we analyzed all the

2 possible parameters combination and achieved the global minimum solution.

3 This 'fit' was based on the 'least squares' method and minimizes the residual

$4 \quad r=\sum_{i=1}^{n}\left(y_{i}-y_{i}^{\prime}\right)^{2}$ where, $y_{i}$ and $y_{i}{ }^{\prime}$ stand for the observed values and the

5 fitted ones, respectively. The logistic equation (Eq. (24)):

$$
f(s)=\left\langle N_{\max }\right\rangle \frac{1+b e^{-\frac{s}{c}}}{1+d e^{-\frac{s}{c}}}
$$

7 was then fitted to find the average 3D cell connectivity, but with a series of

8 constraints on the parameters (as explained above): $\langle f(s=1)\rangle=6, c\rangle$

$90, \quad d<0,\left\langle N_{\max }\right\rangle \geq 0, d>b$ and if $d<-1$ then $c \ln (-d)<1$. The goodness

10 of fitting was estimated by means of the coefficient of determination, $R^{2}$.

\section{Data availability}

13 All the necessary material to reproduce this study is available at the Center for

14 Open Science repository: https://osf.io/nd5t6.

15

16 


\section{REFERENCES}

Aldaz S, Escudero LM \& Freeman M (2013) Dual role of myosin II during Drosophila imaginal disc metamorphosis. Nat. Commun. 4: 1761

Alt S, Ganguly P \& Salbreux G (2017) Vertex models: from cell mechanics to tissue morphogenesis. Philos. Trans. R. Soc. B Biol. Sci. 372: 20150520

Ambrosini A, Gracia M, Proag A, Rayer M, Monier B \& Suzanne M (2017) Apoptotic forces in tissue morphogenesis. Mech. Dev. 144: 33-42

Arganda-Carreras I, Kaynig V, Rueden C, Eliceiri KW, Schindelin J, Cardona A \& Sebastian Seung H (2017) Trainable Weka Segmentation: a machine learning tool for microscopy pixel classification. Bioinformatics 33: 24242426

Bassel GW, Stamm P, Mosca G, Barbier de Reuille P, Gibbs DJ, Winter R, Janka A, Holdsworth MJ \& Smith RS (2014) Mechanical constraints imposed by 3D cellular geometry and arrangement modulate growth patterns in the Arabidopsis embryo. Proc. Natl. Acad. Sci. 111: 86858690

Bertet C, Sulak L \& Lecuit T (2004) Myosin-dependent junction remodelling controls planar cell intercalation and axis elongation. Nature 429: 667671

Bi D, Lopez JH, Schwarz JM \& Lisa Manning M (2014) Energy barriers and cell migration in densely packed tissues. Soft Matter 10: 1885-1890

Bi D, Lopez JH, Schwarz JM \& Manning ML (2015) A density-independent rigidity transition in biological tissues. Nat. Phys. 11: 1074-1079

Bonnefont X, Lacampagne A, Sanchez-Hormigo A, Fino E, Creff A, Mathieu M-N, Smallwood S, Carmignac D, Fontanaud P, Travo P, Alonso G, Courtois-Coutry N, Pincus SM, Robinson ICAF \& Mollard P (2005) Revealing the large-scale network organization of growth hormonesecreting cells. Proc. Natl. Acad. Sci. 102: 16880-16885

Campàs $O$, Mammoto $T$, Hasso $S$, Sperling RA, O'Connell D, Bischof AG, Maas R, Weitz DA, Mahadevan L \& Ingber DE (2014) Quantifying cellgenerated mechanical forces within living embryonic tissues. Nat. Methods 11: 183-189

Canela-Xandri O, Sagués F, Casademunt J \& Buceta J (2011) Dynamics and Mechanical Stability of the Developing Dorsoventral Organizer of the Wing Imaginal Disc. PLoS Comput. Biol. 7: e1002153

Clevers H (2016) Modeling Development and Disease with Organoids. Cell 165: 1586-1597

Colas J-F \& Schoenwolf GC (2001) Towards a cellular and molecular understanding of neurulation. Dev. Dyn. 221: 117-145

Curran S, Strandkvist C, Bathmann J, de Gennes M, Kabla A, Salbreux G \& Baum B (2017) Myosin II Controls Junction Fluctuations to Guide Epithelial Tissue Ordering. Dev. Cell 43: 480-492.e6

Elowitz MB (2002) Stochastic Gene Expression in a Single Cell. Science (80-. ). 297: 1183-1186

Farhadifar R, Röper J-C, Aigouy B, Eaton S \& Jülicher F (2007) The Influence of Cell Mechanics, Cell-Cell Interactions, and Proliferation on Epithelial 
Packing. Curr. Biol. 17: 2095-2104

Fletcher AG, Osterfield M, Baker RE \& Shvartsman SY (2014) Vertex models of epithelial morphogenesis. Biophys. J. 106: 2291-2304

Gibson MC, Patel AB, Nagpal R \& Perrimon N (2006) The emergence of geometric order in proliferating metazoan epithelia. Nature 442: 10381041

Gibson WT, Veldhuis JH, Rubinstein B, Cartwright HN, Perrimon N, Brodland GW, Nagpal R \& Gibson MC (2011) Control of the mitotic cleavage plane by local epithelial topology. Cell 144: 427-438

Gilbert SF \& Barresi MJF (2016) Developmental biology 11th ed. Sunderland, MA Sinauer Associates

Girdler GC \& Röper K (2014) Controlling cell shape changes during salivary gland tube formation in Drosophila. Semin. Cell Dev. Biol. 31: 74-81

Gómez-Gálvez P, Vicente-Munuera P, Tagua A, Forja C, Castro AMAM, Letrán $M$, Valencia-Expósito $A$, Grima $C$, Bermúdez-Gallardo M, SerranoPérez-Higueras Ó, Cavodeassi F, Sotillos S, Martín-Bermudo MDMD, Márquez A, Buceta J \& Escudero LM (2018) Scutoids are a geometrical solution to three-dimensional packing of epithelia. Nat. Commun. 9: 2960

Heller D, Hoppe A, Restrepo S, Gatti L, Tournier AL, Tapon N, Basler K \& Mao Y (2016) EpiTools: An Open-Source Image Analysis Toolkit for Quantifying Epithelial Growth Dynamics. Dev Cell 36: 103-116

Hirashima T \& Adachi T (2019) Polarized cellular mechano-response system for maintaining radial size in developing epithelial tubes. Development 146: $\operatorname{dev} 181206$

Honda H (1978) Description of cellular patterns by Dirichlet domains: the twodimensional case. J Theor Biol 72: 523-543

Huch M, Knoblich JA, Lutolf MP \& Martinez-Arias A (2017) The hope and the hype of organoid research. Dev. 144: 938-941

Huebner RJ \& Ewald AJ (2014) Cellular foundations of mammary tubulogenesis. Semin. Cell Dev. Biol. 31: 124-131

Inoue $\mathrm{Y}$, Tateo I \& Adachi T (2019) Epithelial tissue folding pattern in confined geometry. Biomech. Model. Mechanobiol.

Iruela-Arispe ML \& Beitel GJ (2013) Tubulogenesis. Development 140: 28512855

Irvine KD \& Wieschaus E (1994) Cell intercalation suring Drosophila germband extension and its regulaton by pair00rule segmentation genes. Development 120: 827-841

Khan Z, Wang Y-C, Wieschaus EF \& Kaschube M (2014) Quantitative 4D analyses of epithelial folding during Drosophila gastrulation. Development 141: 2895-2900

Latorre E, Kale S, Casares L, Gómez-González M, Uroz M, Valon L, Nair R V., Garreta E, Montserrat N, del Campo A, Ladoux B, Arroyo M \& Trepat X (2018) Active superelasticity in three-dimensional epithelia of controlled shape. Nature 563: 203-208

Leptin M \& Grunewald B (1990) Cell shape changes during gastrulation in Drosophila. Development 110: 73-84 
Lewis FT (1928) The correlation between cell division and the shapes and sizes of prismatic cells in the epidermis of cucumis. Anatom. Rec. 38: 341-376

Lloyd S (1982) Least squares quantization in PCM. IEEE Trans. Inf. Theory 28: $129-137$

Machado S, Mercier V \& Chiaruttini N (2019) LimeSeg: a coarse-grained lipid membrane simulation for 3D image segmentation. BMC Bioinformatics 20: 2

Mao Y, Tournier AL, Hoppe A, Kester L, Thompson BJ \& Tapon N (2013) Differential proliferation rates generate patterns of mechanical tension that orient tissue growth. EMBO J. 32: 2790-2803

Messal HA, Alt S, Ferreira RMM, Gribben C, Wang VM-Y, Cotoi CG, Salbreux $G$ \& Behrens A (2019) Tissue curvature and apicobasal mechanical tension imbalance instruct cancer morphogenesis. Nature 566: 126

Misra M, Audoly B \& Shvartsman SY (2017) Complex structures from patterned cell sheets. Philos. Trans. R. Soc. B Biol. Sci. 372: 20150515

Mongera A, Rowghanian P, Gustafson HJ, Shelton E, Kealhofer DA, Carn EK, Serwane F, Lucio AA, Giammona J \& Campàs O (2018) A fluid-to-solid jamming transition underlies vertebrate body axis elongation. Nature 561: 401-405

Mughal A, Cox SJ, Weaire D, Burke SR \& Hutzler S (2018) Demonstration and interpretation of 'scutoid' cells formed in a quasi-2D soap froth. Philos. Mag. Lett. 98: 358-364

Nelson CM (2009) Geometric control of tissue morphogenesis. Biochim. Biophys. Acta - Mol. Cell Res. 1793: 903-910

Nelson CM, Jean RP, Tan JL, Liu WF, Sniadecki NJ, Spector AA \& Chen CS (2005) Emergent patterns of growth controlled by multicellular form and mechanics. Proc. Natl. Acad. Sci. 102: 11594-11599

Okuda S, Kuranaga E \& Sato K (2019) Apical Junctional Fluctuations Lead to Cell Flow while Maintaining Epithelial Integrity. Biophys. J. 116: 11591170

Pérez-González C, Alert R, Blanch-Mercader C, Gómez-González M, Kolodziej T, Bazellieres E, Casademunt J \& Trepat X (2019) Active wetting of epithelial tissues. Nat. Phys. 15: 79-88

Pilot F \& Lecuit T (2005) Compartmentalized morphogenesis in epithelia: from cell to tissue shape. Dev Dyn 232: 685-694

Reinhardt K (1918) Über die Zerlegung der Ebene in Polygone.

Röper K (2018) Quantitative Imaging and the Effect of Tissue Topology on Morphogenesis. Dev. Cell 47: 537-538

Rupprecht JF, Ong KH, Yin J, Huang A, Dinh HHQ, Singh AP, Zhang S, Yu W \& Saunders TE (2017) Geometric constraints alter cell arrangements within curved epithelial tissues. Mol. Biol. Cell 28: 3582-3594

Sanchez-Corrales YE, Blanchard GB \& Röper K (2018) Radially patterned cell behaviours during tube budding from an epithelium. Elife 7: e35717

Sánchez-Gutiérrez D, Tozluoglu M, Barry JD, Pascual A, Mao Y \& Escudero LM (2016) Fundamental physical cellular constraints drive selforganization of tissues. EMBO J. 35: 77-88 
Schindelin J, Arganda-Carreras I, Frise E, Kaynig V, Longair M, Pietzsch T, Preibisch S, Rueden C, Saalfeld S, Schmid B, Tinevez J-Y, White DJ, Hartenstein V, Eliceiri K, Tomancak P \& Cardona A (2012) Fiji: an opensource platform for biological-image analysis. Nat. Methods 9: 676-682

Schutgens F, Rookmaaker MB, Margaritis T, Rios A, Ammerlaan C, Jansen J, Gijzen L, Vormann M, Vonk A, Viveen M, Yengej FY, Derakhshan S, de Winter-de Groot KM, Artegiani B, van Boxtel R, Cuppen E, Hendrickx APA, van den Heuvel-Eibrink MM, Heitzer E, Lanz H, et al (2019) Tubuloids derived from human adult kidney and urine for personalized disease modeling. Nat. Biotechnol. 37: 303-313

Shahbazi MN, Siggia ED \& Zernicka-Goetz M (2019) Self-organization of stem cells into embryos: A window on early mammalian development. Science (80-. ). 364: 948-951

Sharma P, Saraswathy VM, Xiang L \& Furthauer M (2019) Delta/Notch signaling controls neuroepithelial morphogenesis in the zebrafish spinal cord. bioRxiv: 517714

Siedlik MJ, Manivannan S, Kevrekidis IG \& Nelson CM (2017) Cell Division Induces and Switches Coherent Angular Motion within Bounded Cellular Collectives. Biophys. J. 112: 2419-2427

Spencer MA, Jabeen Z \& Lubensky DK (2017) Vertex stability and topological transitions in vertex models of foams and epithelia. Eur. Phys. J. E 40: 2

Sugimura K, Lenne PF \& Graner F (2016) Measuring forces and stresses in situ in living tissues. Development 143: 186-196

Swanson LE \& Beitel GJ (2006) Tubulogenesis: an inside job. Curr Biol 16: R51-3

Tetley RJ, Staddon MF, Heller D, Hoppe A, Banerjee S \& Mao Y (2019) Tissue fluidity promotes epithelial wound healing. Nat. Phys. 15: 11951203

Thompson DWD (1945) On growth and form Cambridge university press

Trepat X, Wasserman MR, Angelini TE, Millet E, Weitz DA, Butler JP \& Fredberg JJ (2009) Physical forces during collective cell migration. Nat. Phys. 5: 426-430

Tung JJ, Tattersall IW \& Kitajewski J (2012) Tips, Stalks, Tubes: NotchMediated Cell Fate Determination and Mechanisms of Tubulogenesis during Angiogenesis. Cold Spring Harb. Perspect. Med. 2: a006601a006601

Wetzel G (1926) Zur entwicklungsmechanischen Analyse des einfachen prismatischen Epithels. Wilhelm Roux Arch. für Entwicklungsmechanik der Org. 107: 177-185

Yang R, Li E, Kwon Y-J, Mani M \& Beitel GJ (2019) QuBiT: a quantitative tool for analyzing epithelial tubes reveals unexpected patterns of organization in the Drosophila trachea. Development 146: dev172759

Yang X, Bi D, Czajkowski M, Merkel M, Manning ML \& Marchetti MC (2017) Correlating cell shape and cellular stress in motile confluent tissues. Proc. Natl. Acad. Sci. 114: 12663-12668 
1 Figure 1. Analysis of apico-basal cell intercalations in the Voronoi 2 tubular model

3 a) Scutoids (left) entail apico-basal intercalations among packing cells that can

4 be envisioned as spatial T1 transitions to exchange neighbors (right). The

5 green and the red cells are neighbors in basal (but not in apical) while the

6 opposite is true for the blue and the yellow cells. b) Voronoi in silico tubes with

7 different surface ratios, $s_{b}: s_{b}=2$ indigo blue; $s_{b}=5$, dark blue (apical

8 surface, light blue). c) For a given radial section (plane), cell boundaries

9 emerge by applying a Voronoi tessellation to a number of seeds located in the

10 plane. In the V1 (Voronoi 1) model seeds are randomly distributed. By

11 applying iteratively the Lloyd algorithm (left to right) the topological disorder

12 diminishes (Materials and Methods). d) The density plot shows the average

13 number of apico-basal intercalations per cell in in silico tubes $(n=20)$ as a

14 function of the surface ratio and the Voronoi class. 
1 Figure 2. Three-dimensional packing and connectivity properties of the 2 Voronoi tubular model

3 a) A schematic representation of a $3 D$ histogram (density plot) where all cells

4 have prismatic-like shapes (i.e., in the absence of scutoids). The histogram

5 accounts for the probability that cells have $n_{a}$ (number) of neighbors in the

6 apical surface and $n_{b}$ neighbors in the basal surface. If there are no scutoids,

7 there are only contributions in the diagonal bins whereas if there are scutoids

8 the distribution spreads away from the diagonal. b) 3D histograms of V5 tubes

9 for increasing values of the surface ratio. The larger value of the spreading

10 coefficient, $\eta^{2}$, (Material and Methods) indicates an increasing number of

11 scutoids. c) and d) Density plots showing $\eta^{2}$ (c) and the average number of

$123 \mathrm{D}$ neighbors, $\left\langle n_{3 D}\right\rangle$, (d) as a function of the surface ratio and the Voronoi

13 class in in silico tubes $(n=20)$. 


\section{Figure 3. Cells in the Voronoi tubular model follow a "poor get richer"}

2 principle

3 a) Average net gain of neighbors (density plot) with respect to the apical

4 surface in Voronoi tubes with a surface ratio $s_{b}=5$ as a function of the

5 Voronoi class and the apical polygonal class $(n=20)$. Cells with a smaller

6 polygonal class are more prone to gain neighbors. b) "Poor get richer"

7 principle in V5 tubes with a surface ratio $s_{b}=5$. The size of the circle accounts

8 for the relative data count within each apical polygon class (numbers indicate

9 the number of cells that gained 3D neighbors). The boxes indicate the

$1025 \%-75 \%$ percentile interval, black lines the mean values, gray lines the

11 standard deviation, and the red dotted lines the statistical median. 
1 Box. In tubular geometries the 3D cellular connectivity gain decreases as

2 the surface ratio increases and it is bounded. In this example, the $Y$ axis

3 represents the longitudinal axis of tubes, while the $X$ axis accounts for the

4 Cartesian projection of the transversal axis of radial sections. From left to right

5 different radial sections are represented as $s$ increases (as indicated by the

6 color gradient arrow: from light to dark blue). In a) three Voronoi seeds that

7 correspond to neighboring cells at the apical surface, $s=1$, define the triangle

$8 A B C$. Panels b) and c) track changes in the neighboring relations

9 (accumulated neighbors) of cell $A$ for two increasing values of $s: 2$ and 4.5

10 (panels $\mathbf{b})$ and $\mathbf{c}$ ) respectively). As shown in a), should a new neighboring cell,

$11 D$, of cell $A$ appear due to an apico-basal intercalation, then its position must

12 lie inside the parabola defined by the points $A, B$ and $C$, but outside the circle

13 that these points define (white region), (see Lemma 1, Material and

14 Methods). Regions accessible to new neighbors are then coded by the green

15 shading in a)-c). As $s$ increases, see $\mathbf{b})$, and the cells $A, B, C$, and $D$ become

16 neighbors, then the parabolas and circles defined by $A B D$ and $A C D$ restrict the

17 locations of possible new neighbors. This idea is further reinforced in panel c):

18 winning neighbor $E$ set additional limits to the accessible locations of new

19 neighbors. Thus, the potentiality of a connectivity gain by cell $A$ due to apico-

20 basal intercalations diminishes as the surface ratio increases and eventually

21 becomes null: the number of 3D neighbors of a cell is bounded (Materials and

\section{Methods).}


1 Figure 4. A probabilistic model reproduces the 3D cell connectivity 2 behavior of epithelial tubes.

3 a) Top: in tubular geometries, cell intercalations along the apico-basal axis can

4 be visualized as non-reversible spatial T1 transitions (once a neighbor is won

5 it cannot be lost). Bottom: the "poor get richer" principle suggests an

6 increasing energetic cost (i.e., a larger activation energy) for recruiting new 3D

7 neighbors. In our model, $\beta$ accounts for the energetic cost per 3D neighbor to

8 recruit a new neighbor (Materials and Methods). b) The energy landscape

9 shown in a) can be modeled by a stochastic dynamics (a Kolmogorov rate

10 equation) where cells increase their 3D neighbors with a probability per unit of

11 surface ratio, $r_{n, m}$, that depends on the activation energy and the maximum

12 cell connectivity $N_{\max }$ (Material and Methods). C) Comparison between

13 results obtained in the Kolmogorov model and simulations of V5 in silico tubes.

14 The left/center density plots represent the connectivity distribution (i.e., the

15 fraction of cells with a given number of $3 D$ neighbors) as a function of the

16 radial expansion obtained in the Voronoi simulation (left) and as predicted by

17 the Kolmogorov model (center); the purple open circles (left/right) indicate the

18 average number of $3 D$ neighbors per cell $\left\langle n_{3 D}\right\rangle$; the solid red line and the

19 dashed white line (center/right) shows $\left\langle n_{3 D}\right\rangle$ as obtained by the Kolmogorov

20 model and the Flinstones' law respectively. The density plot on the right shows

21 the difference between the predicted and the actual connectivity distributions

22 and the corresponding error, $\varepsilon^{2}$ (magenta lines), see Fig. S3 and Material and

\section{Methods.}


1 Figure 5. Packing and connectivity analysis of Drosophila's salivary 2 gland and comparison with the V8 model

3 a) Full projection of a salivary gland (cell contours stained by Cy3-labeled

4 phalloidin, Materials and Methods). b) Computer representation of the

5 segmented salivary gland shown in a) (Material and Methods). c) 3D

6 rendering of a representative segmented salivary gland: apical surface, light

7 green; basal surface, dark green. d) Density plots of the distribution of

8 neighbor exchanges between apical and basal surfaces as a function of the

9 number of neighbors in apical, $n_{a}$, and basal, $n_{b}$, surfaces (as in Fig. 2b):

10 salivary glands (left) and V8 tubes (right) with surface ratio $s_{b}=1.75$. e)

11 Comparison between results obtained in salivary glands (top) and the

12 simulations of the V8 model (bottom) in regards of the 3D cellular connectivity

13 as a function of the surface ratio (see Fig. 4c). 
1 Figure S1. Scutoidal prevalence and relation between $\left\langle n_{3 D}\right\rangle$ and $\langle i\rangle$.

2 a) The density plot shows the percentage of scutoidal cells in the tissue

3 (scutoidal prevalence) as a function of the surface ratio (from $s=1$ to $s=4$

4 with steps of 0.25 ) and the Voronoi class (same samples that in Fig. 1d). We

5 notice that from $s=4$ onward, the scutoidal prevalence in all Voronoi

6 diagrams is $100 \%$. b) Average number of 3D neighbors, $\left\langle n_{3 D}\right\rangle$, as a function of

7 the average number of apico-basal intercalations per cell, $\langle i\rangle$, in Voronoi tubes

8 (from $s=1$ to $s=10$ with steps of $0.5,20$ samples) and in salivary glands

9 (from $s=1$ to $s=3.5$ with steps of $0.5,20$ samples).

11 Figure S2. Poor get richer principle in Voronoi tubes.

12 Average net gain of neighbors (density plot) with respect to the apical surface

13 in Voronoi tubes with surface ratios $s_{b}=1.5$ and $s_{b}=2(\mathbf{a})$, and with respect

14 to the basal surface for $s_{b}=5$ (b) as a function of the Voronoi and the

15 polygonal class $(n=20)$.

17 Figure S3. Comparison between results obtained in the Kolmogorov 18 model and simulations of $\mathrm{V} 1$ and $\mathrm{V} 10$ in silico tubes.

19 As in Fig. 3b, the left/center density plots represent the connectivity

20 distribution (i.e., the fraction of cells with a given number of 3D neighbors) as a

21 function of the radial expansion obtained in the Voronoi simulation (left) and as

22 predicted by the Kolmogorov model (center); the purple open circles (left/right)

23 indicate the average number of $3 D$ neighbors per cell $\left\langle n_{3 D}\right\rangle$; the solid red line

24 and the dashed white line (center/right) shows $\left\langle n_{3 D}\right\rangle$ as obtained by the

25 Kolmogorov model and the Flinstones' law respectively. The density plot on

26 the right shows the difference between the predicted and the actual

27 connectivity distributions and the corresponding error, $\varepsilon^{2}$ (magenta lines).

29 Figure S4. Polygon distribution in apical and basal surfaces and "poor 30 get richer" principle: salivary gland and V8 tubes.

31 a) Polygon distribution of salivary glands and Voronoi 8 (V8) in silico tubes: the

32 error bar accounts for the standard deviation $(n=20)$. b) Average net gain of

33 neighbors (density plot) with respect to the apical surface in salivary glands 
1 (top) and V8 tubes with a surface ratio $s_{b}=1.75$ (middle) and $s_{b}=4$ (bottom)

2 as a function of the apical polygonal class $(n=20)$. c) Absolute net gain of 3D

3 neighbors as a function of the polygonal class in the apical surface (green:

4 salivary gland; blue: V8 tubes). The size of the circle accounts for the relative

5 data count within the apical polygon class (integer numbers indicate the

6 number of cells that gained 3D neighbors). The boxes indicate the $25 \%-75 \%$

7 percentile interval, black lines the mean values, gray lines the standard

8 deviation, and the red dotted lines the statistical median. Cells with a smaller

9 polygonal class are more prone to gain neighbors.

11 Figure S5. Euler characteristic in scutoids.

12 A scutoid solid can be simplified as a collection of vertexes (circles) that can

13 be connected by straight edges. In the figure the green circle accounts of an

14 apico-basal intercalation point. Alternatively, we can represent the solid as a

15 connected plane graph. In both cases the Euler characteristic is 2.

17 Table S1. Fitting parameters (Flintstones' law and Kolmogorov model)

18 and spreading quantification in Voronoi tubes and salivary glands.

19 Tab 1 (Flintstones) shows the independent fitting parameter values

$20\left(c, d,\left\langle N_{\max }\right\rangle\right)$, the values of $b,\left\langle n_{3 D}\right\rangle$ for different, relevant, values of the surface

21 ratio, and the estimated values of $\alpha$ and $\beta$ (Materials and Methods). Tab 2

22 (Kolmogorov) shows the independent fitting parameter values $\left(\alpha, \beta,\left\langle N_{\max }\right\rangle\right)$.

23 Tab 3 (Spreading) shows the spreading values of the 3D histograms of

24 neighbor exchange between basal and apical surfaces (Materials and

25 Methods). 
$\mathbf{a}$

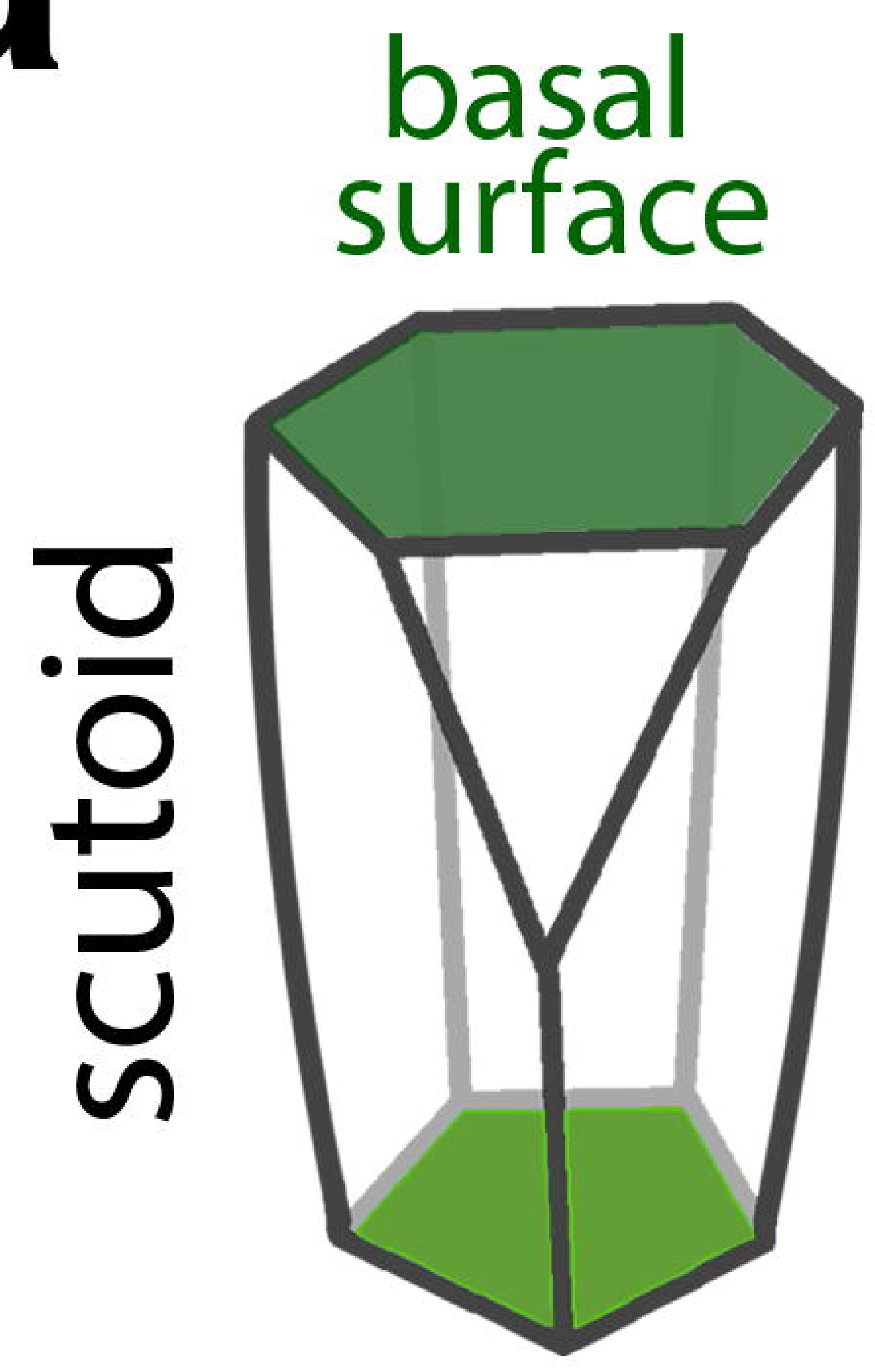

apical

surface
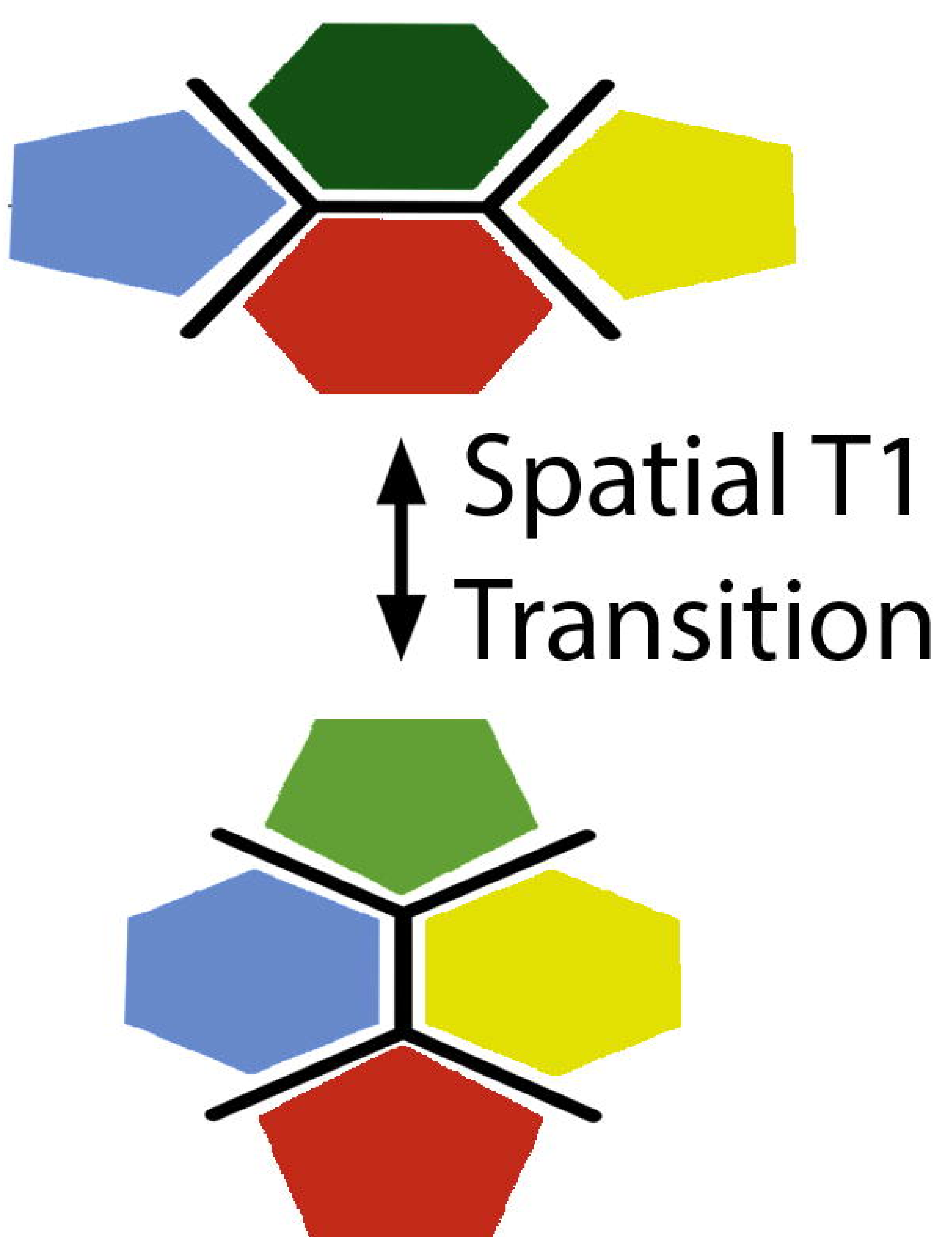

C

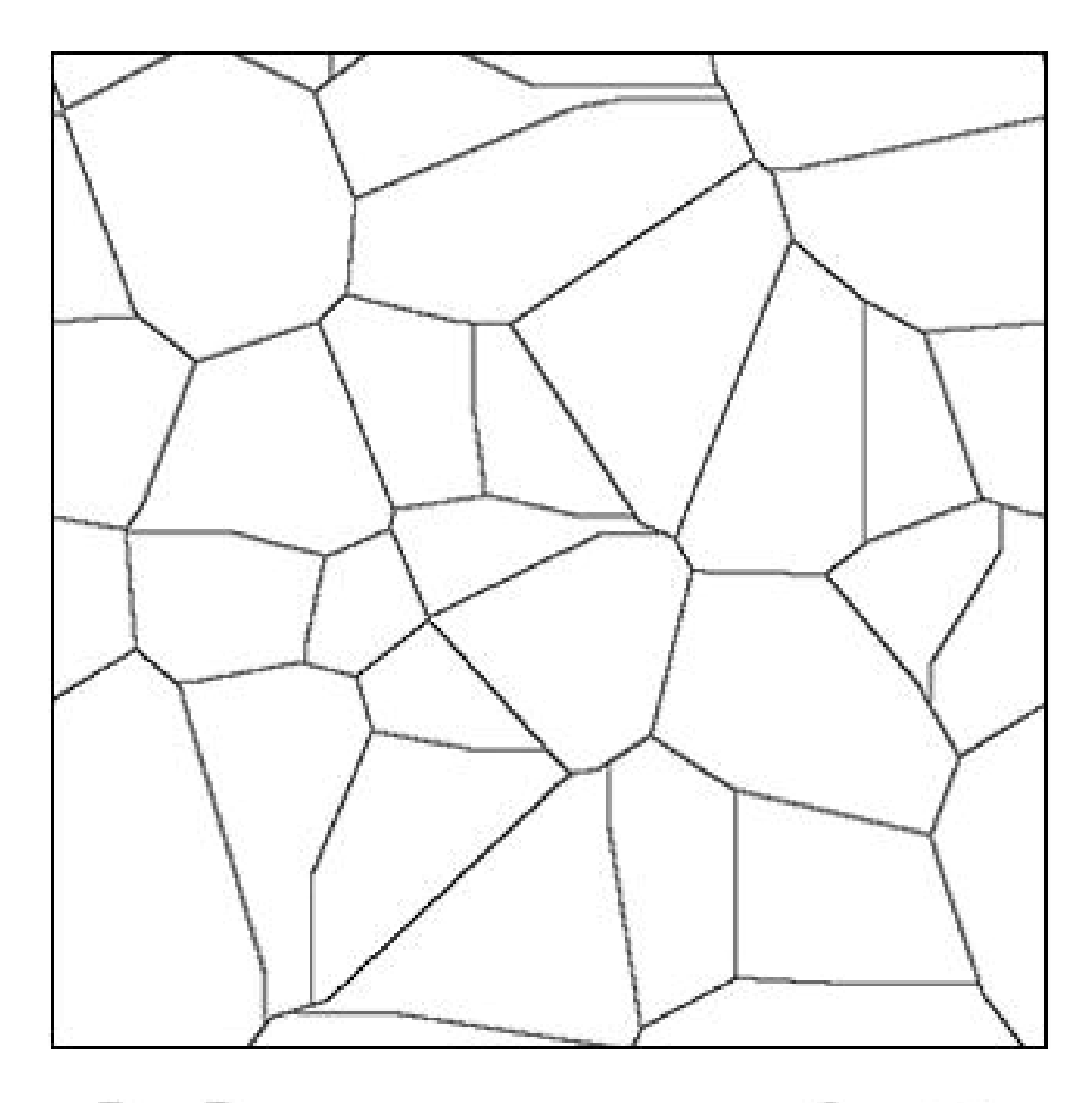

Voronoi 1

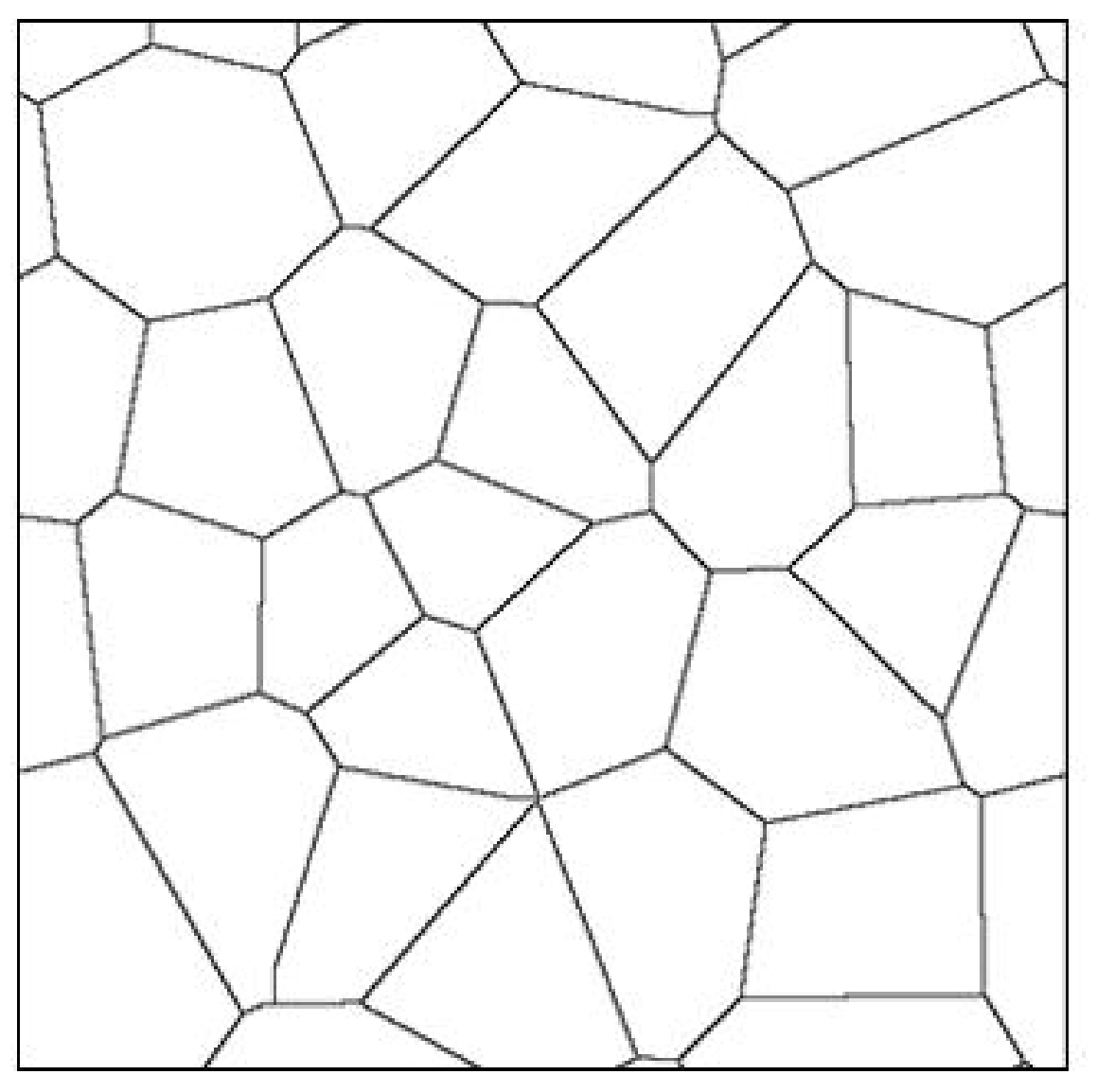

Voronoi 2

b Voronoi tubular model

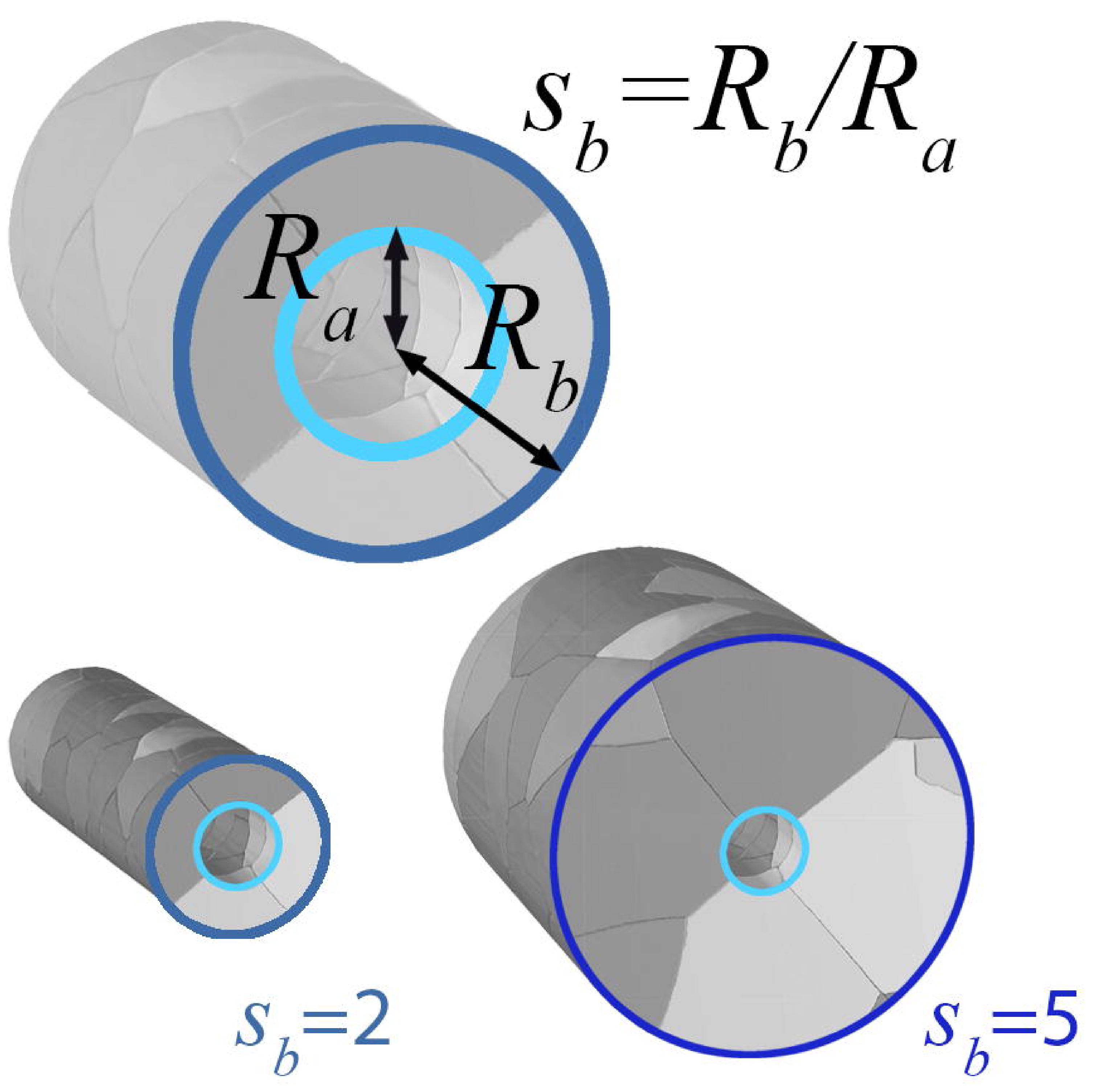

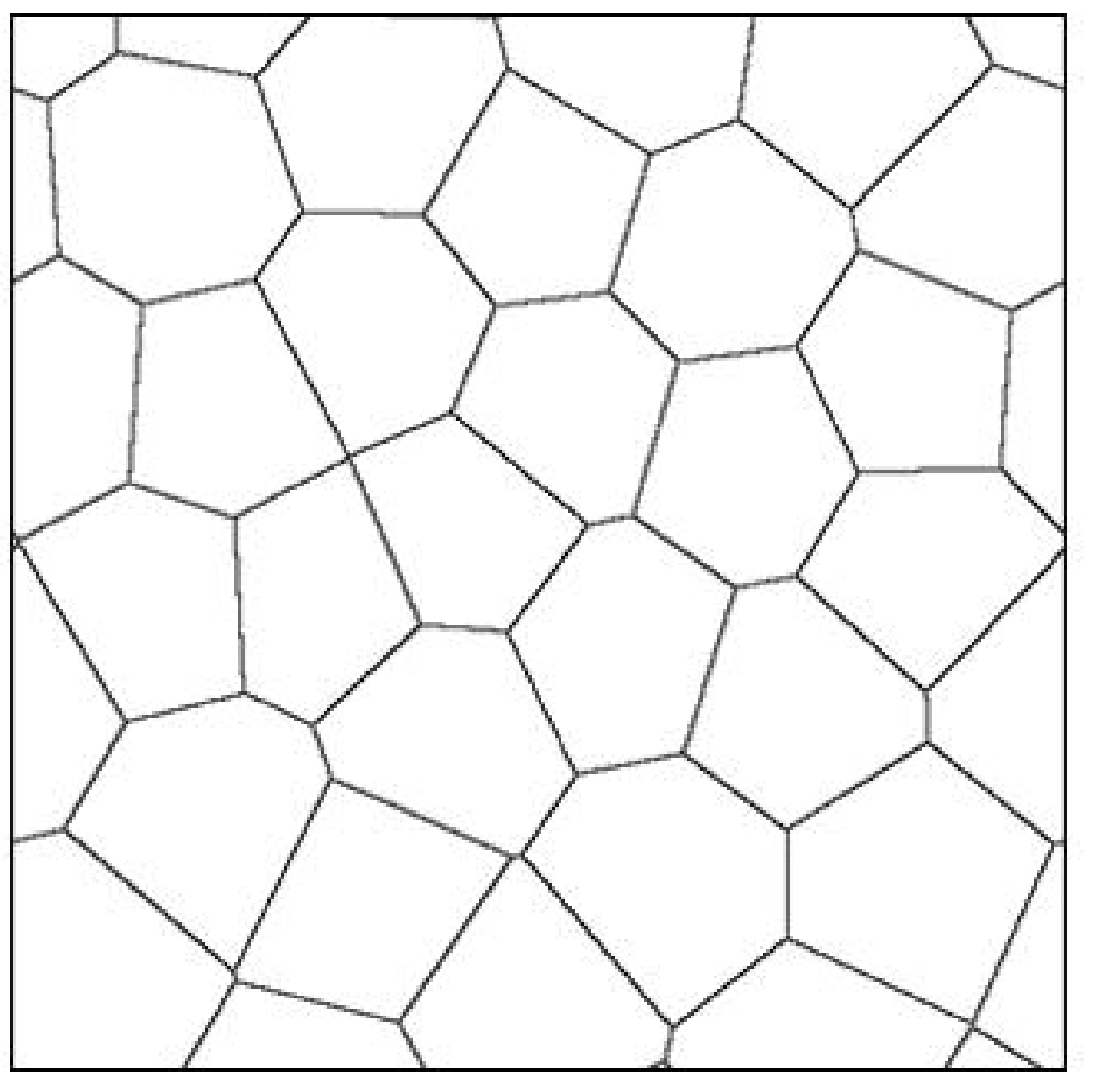

Voronoi 5

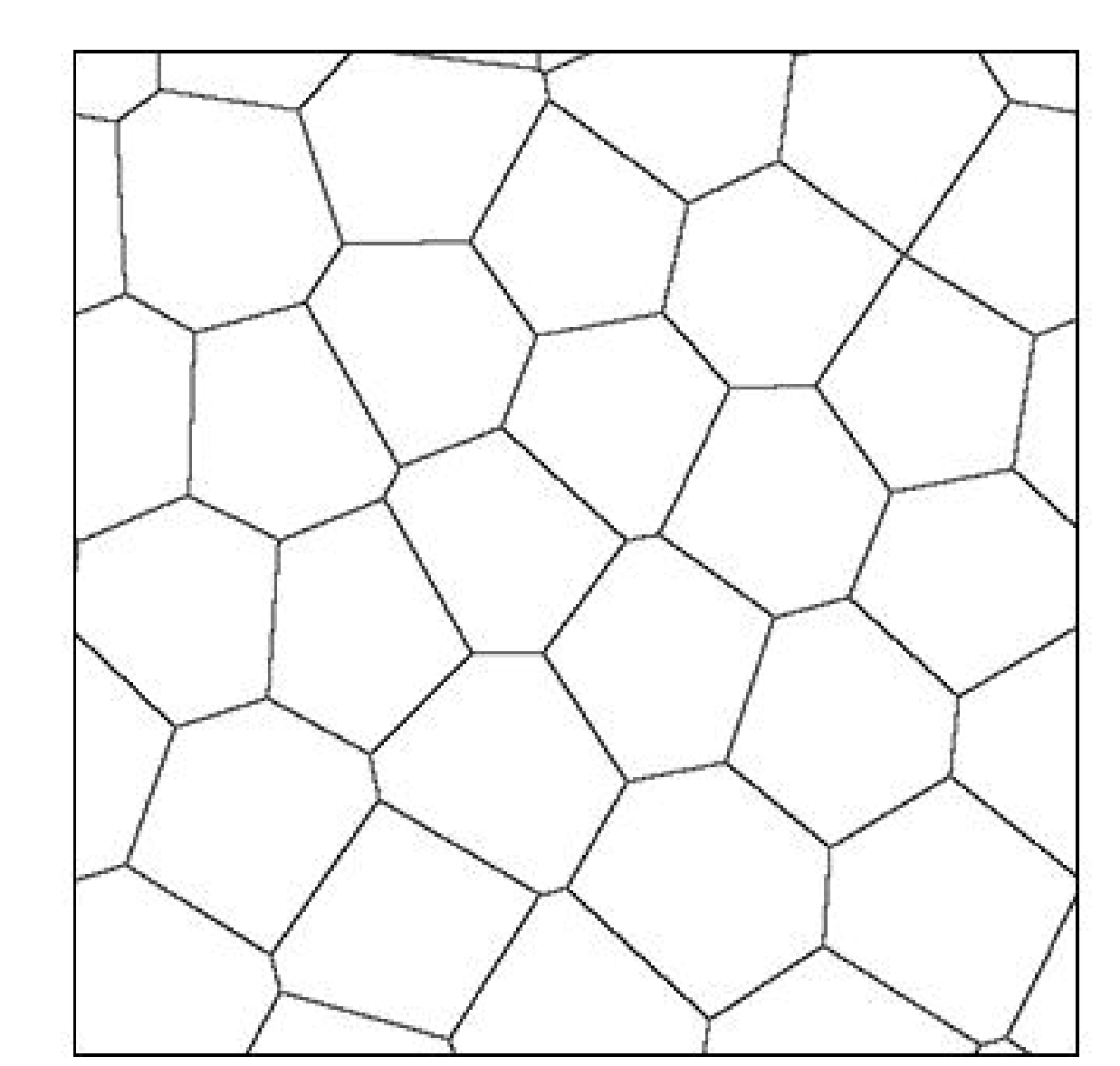

Voronoi 8 Voronoi 10

d

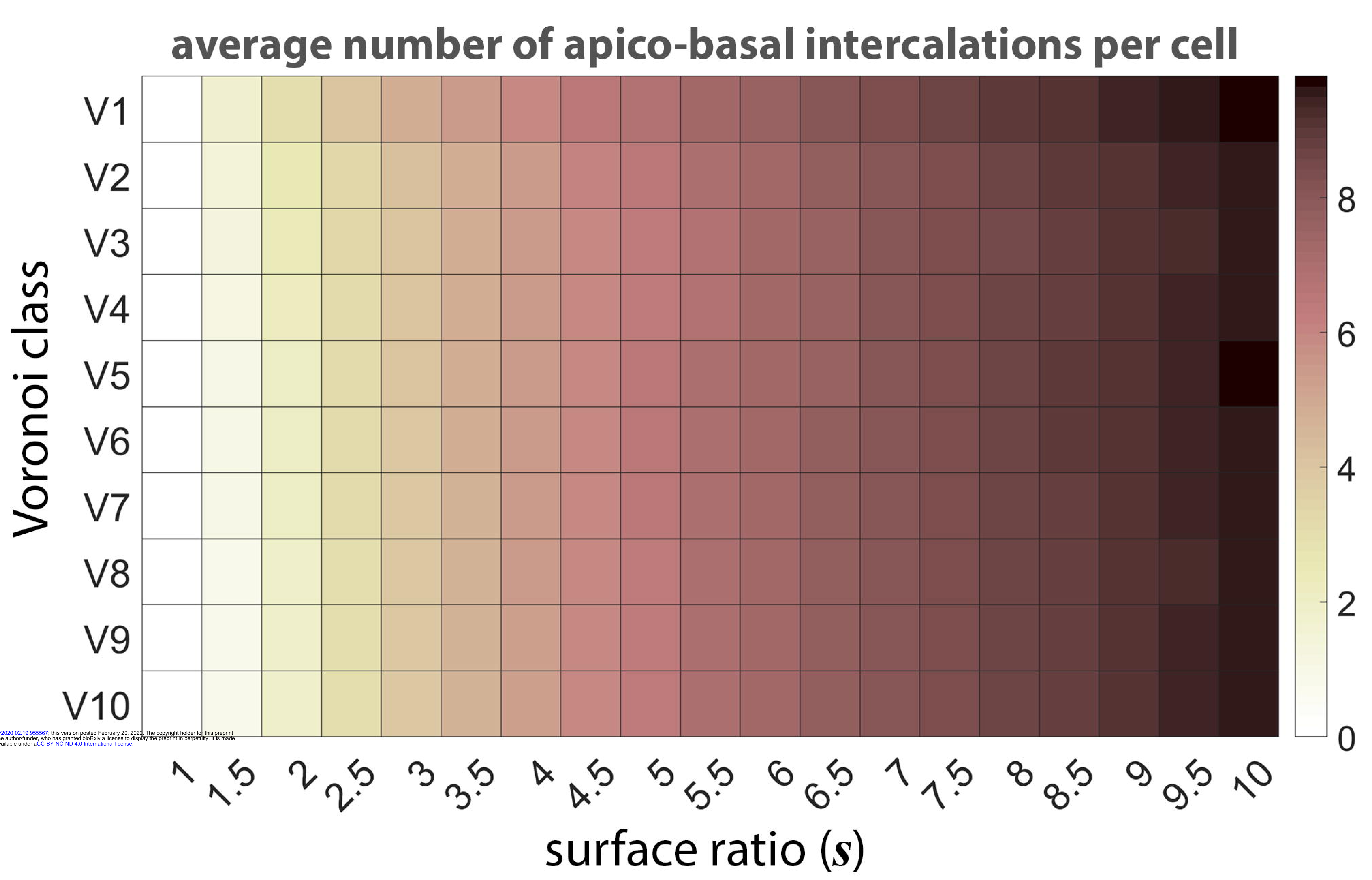



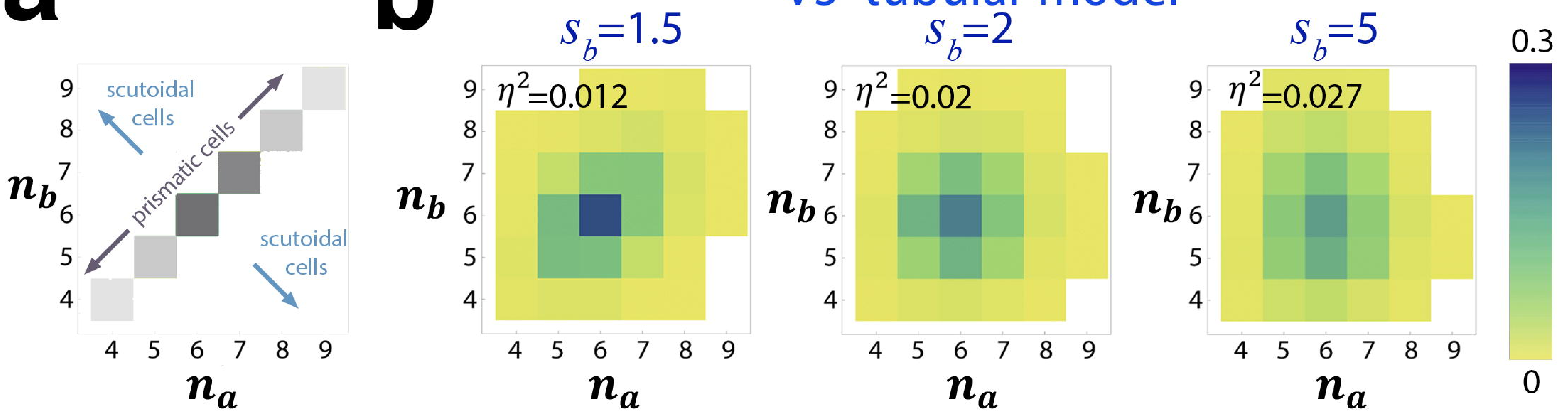

C

$\eta^{2}$

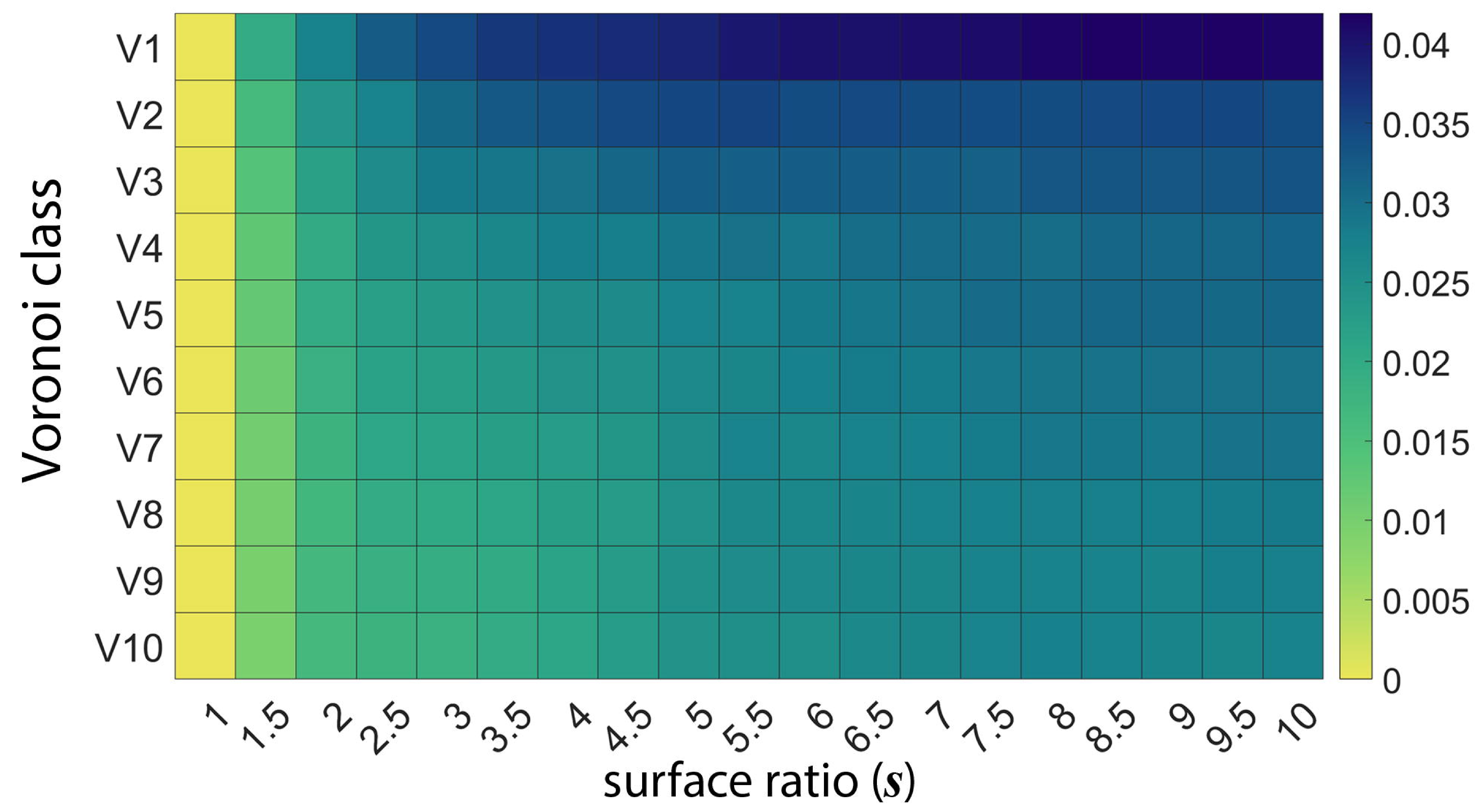

$\left\langle n_{3 D}\right\rangle$

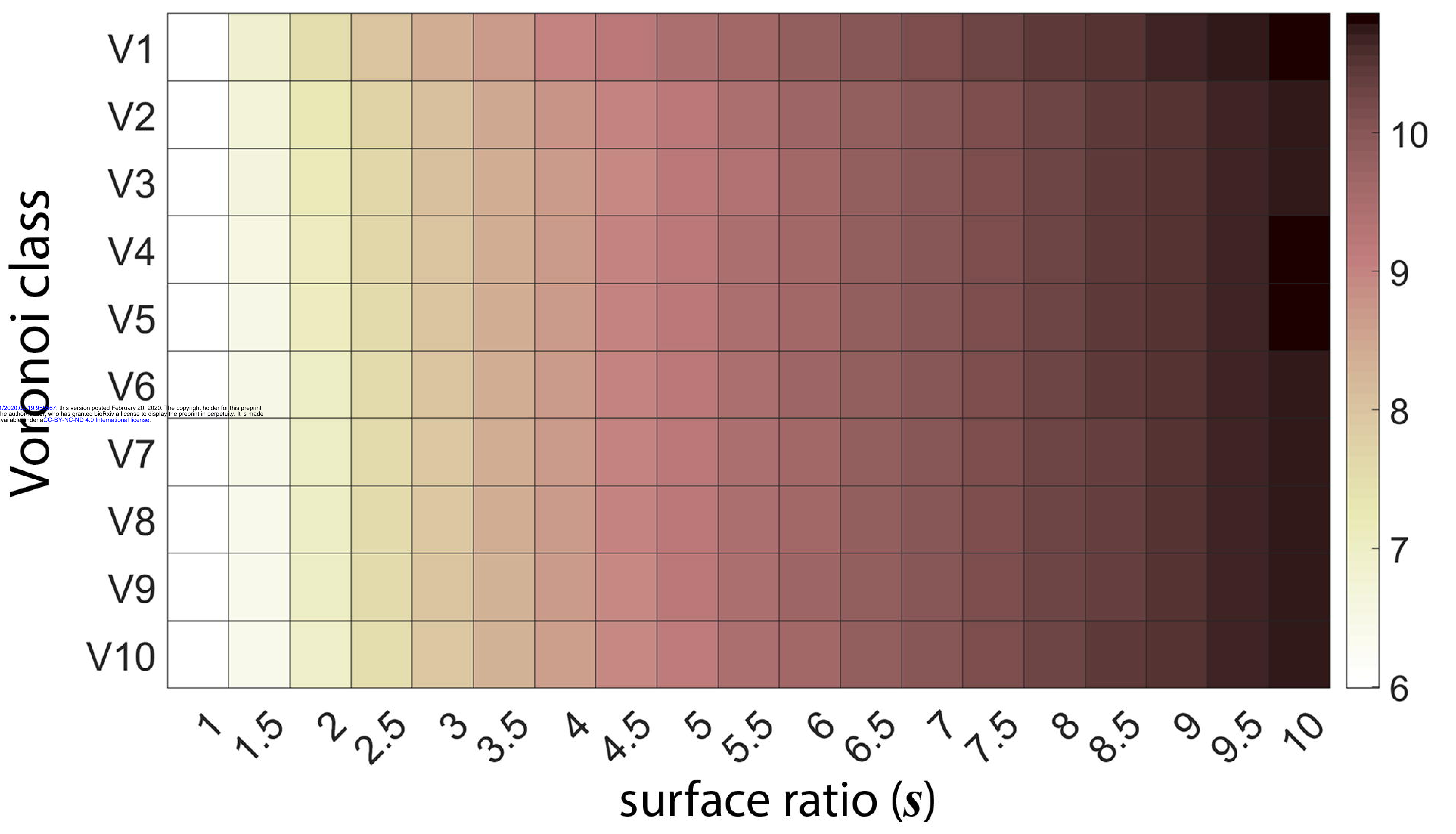


a

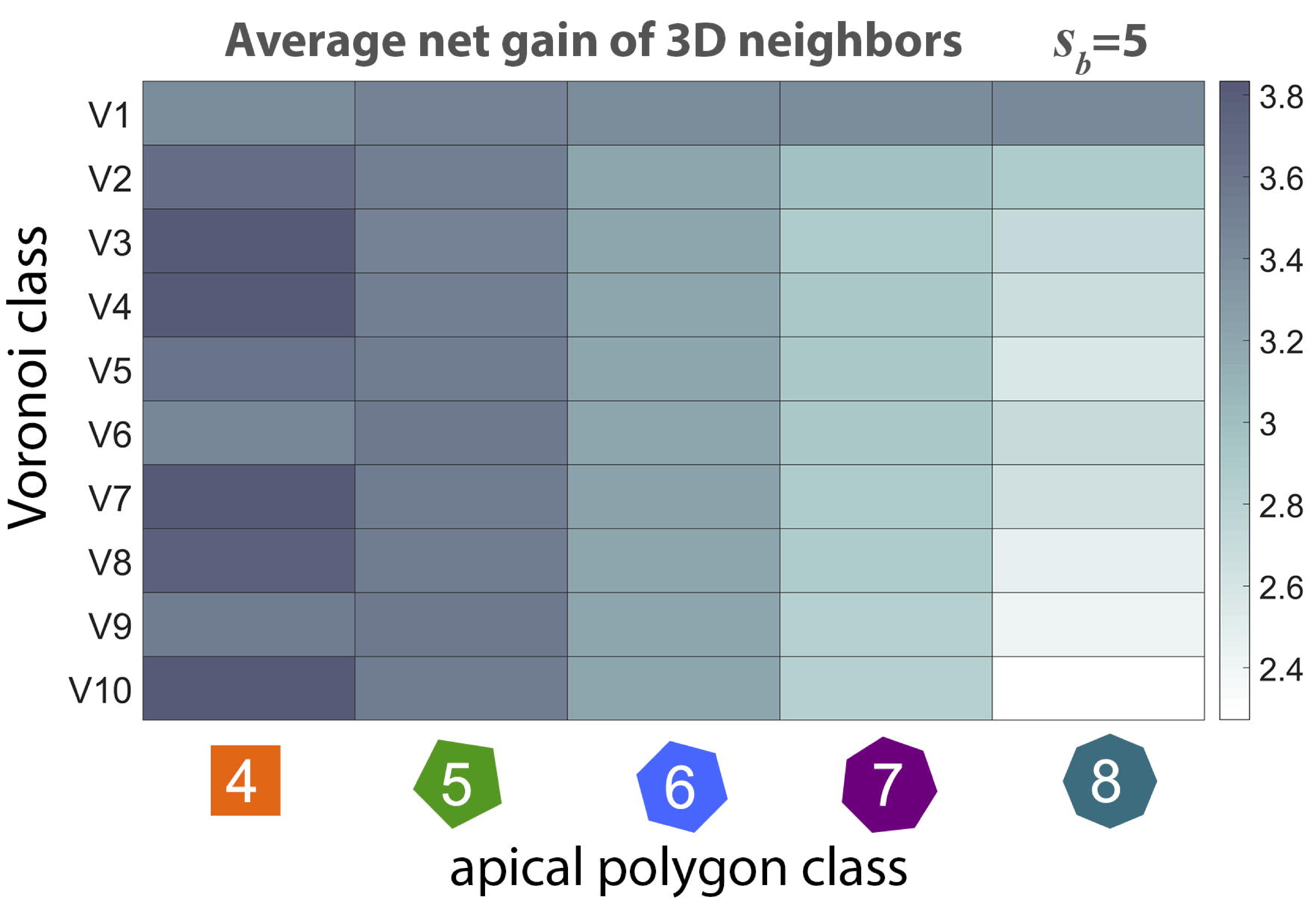

"poor get richer" principle

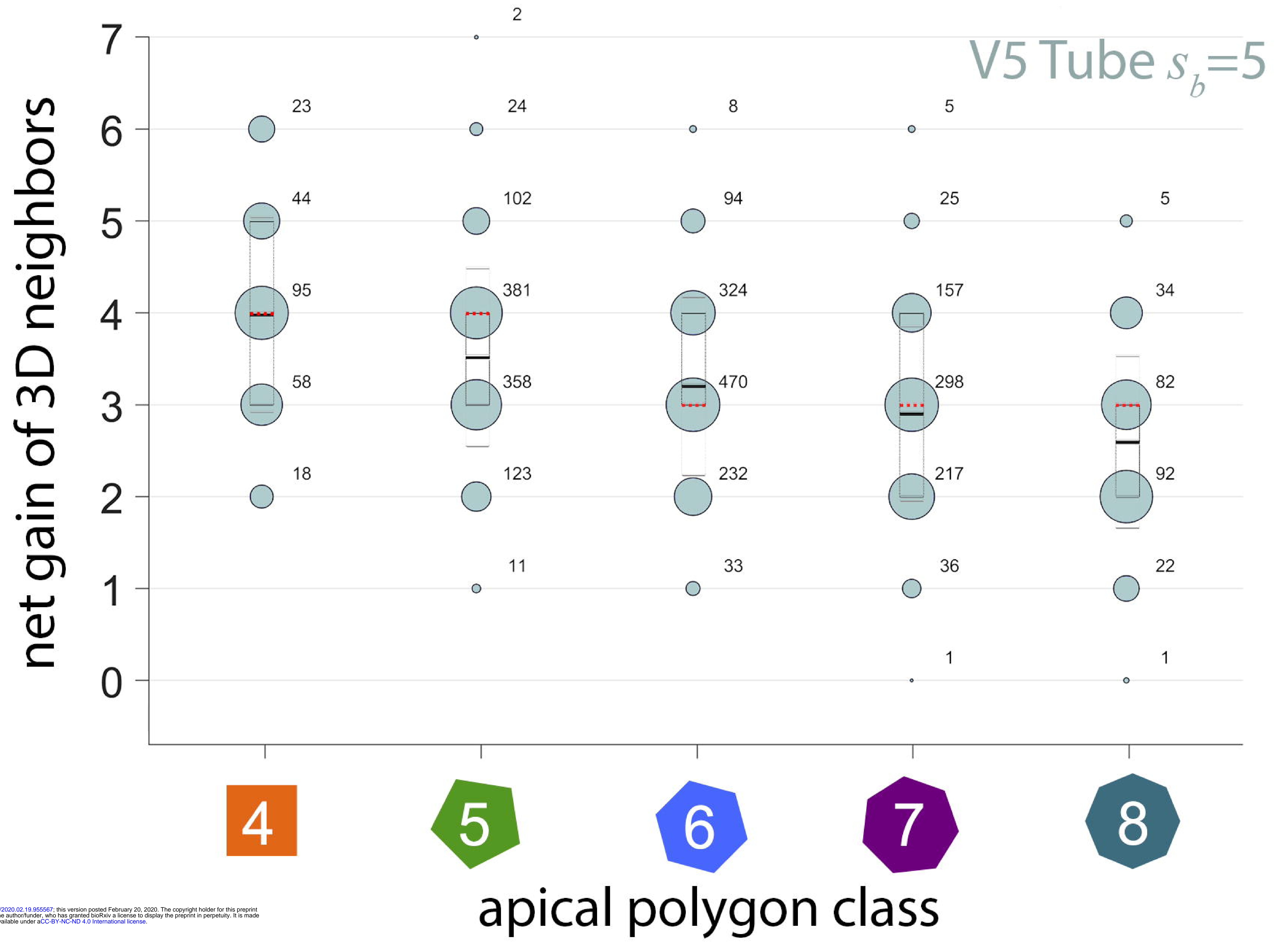




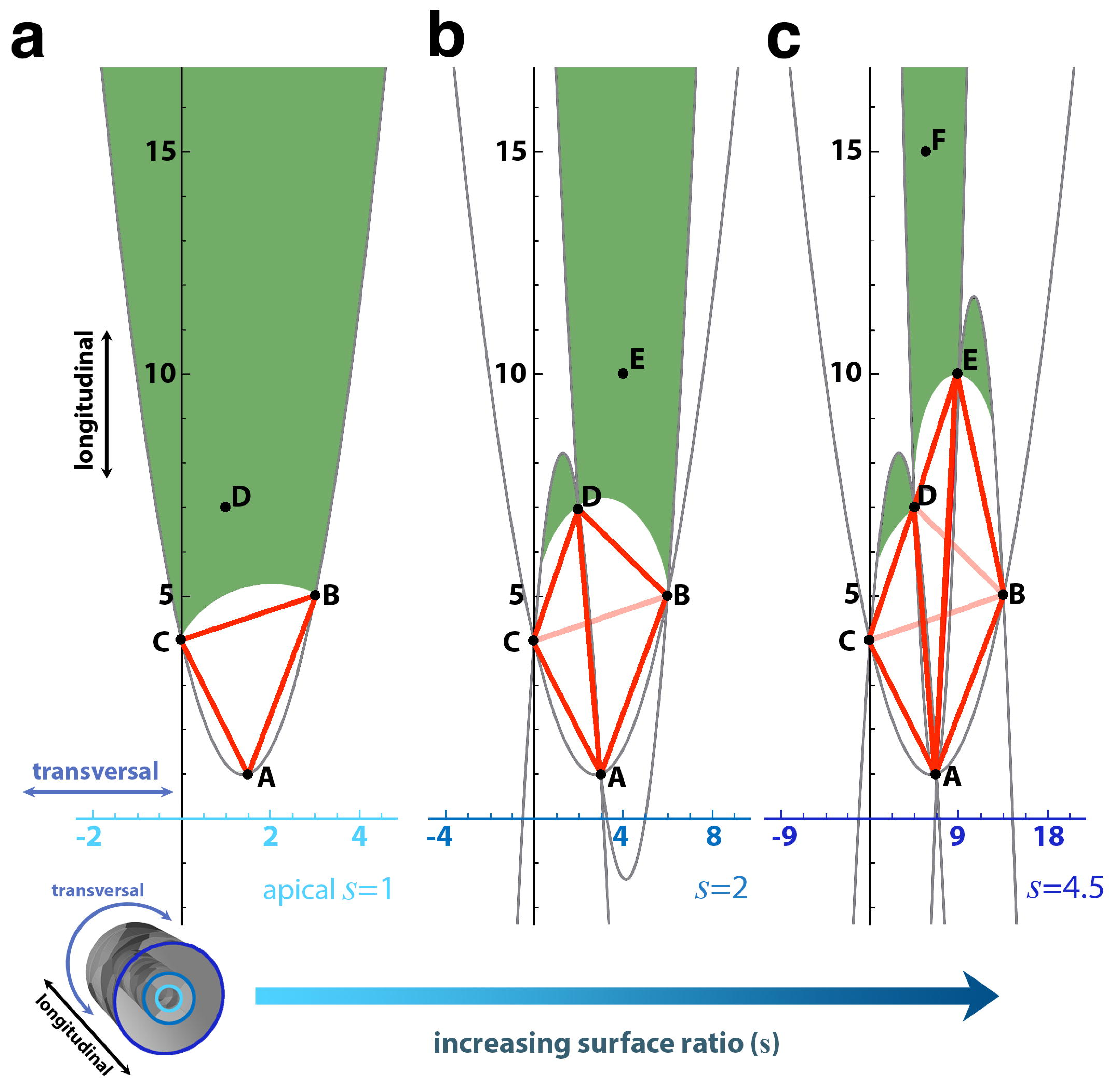


a spatial T1 transition
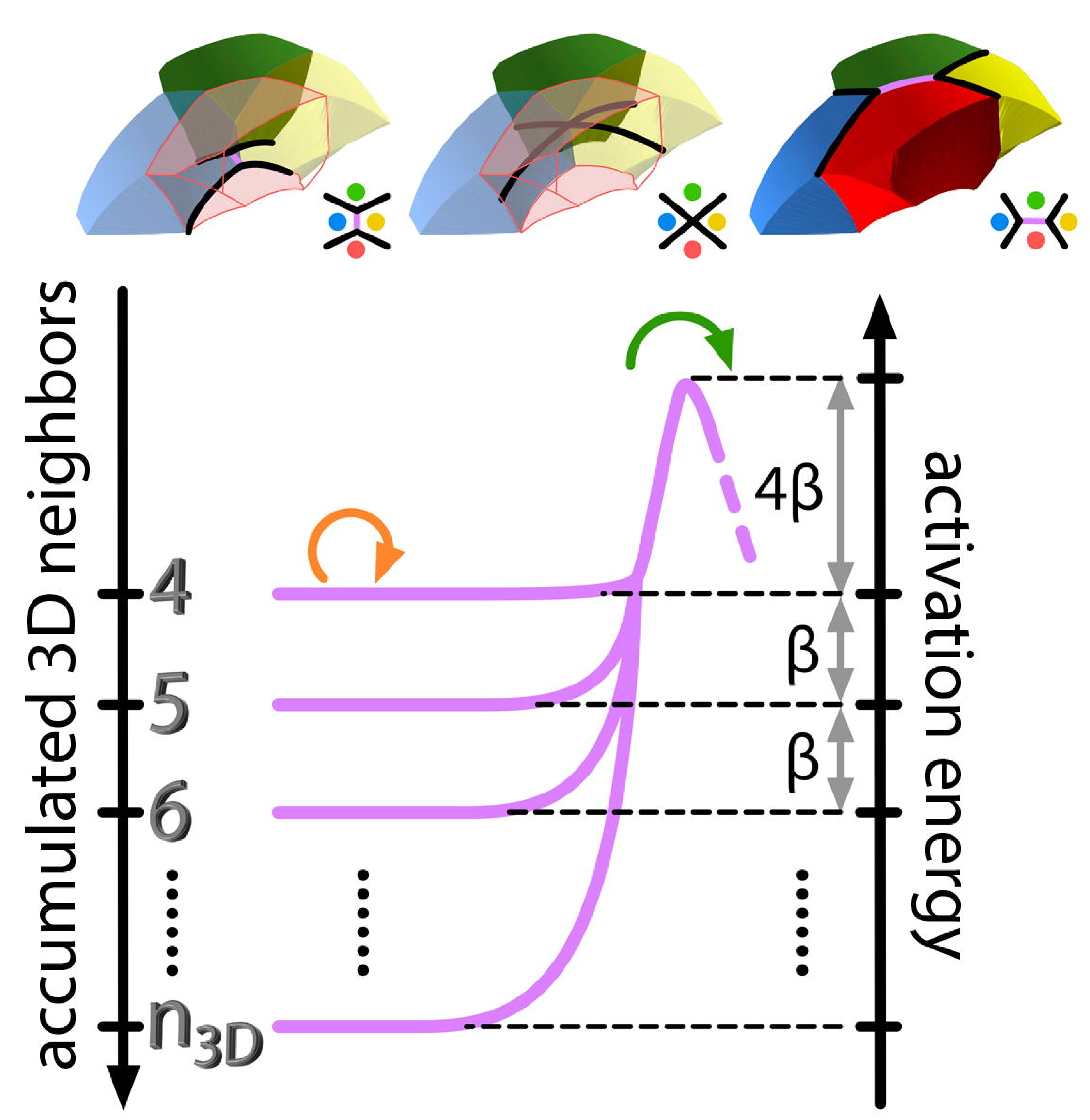

b

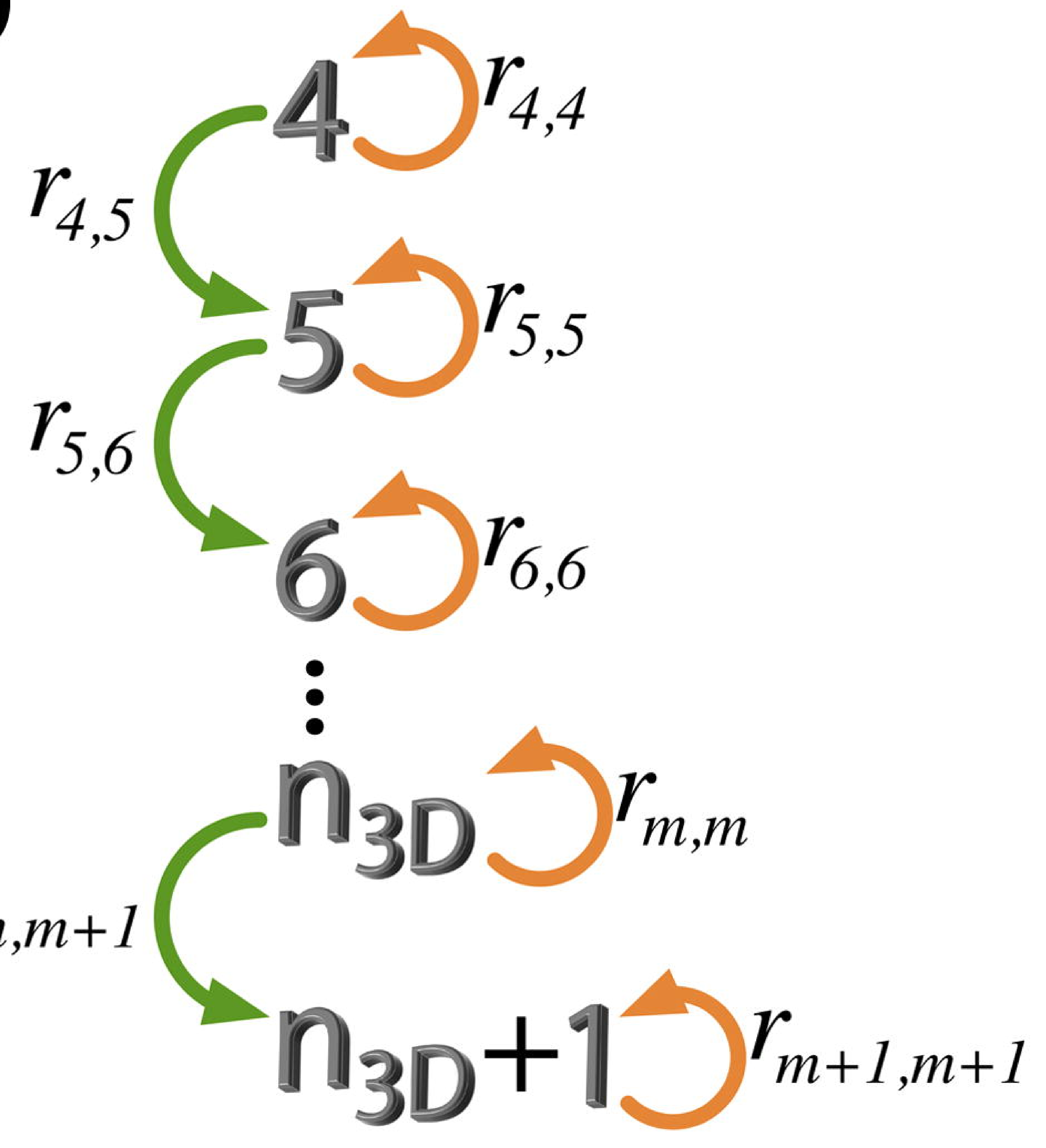

$r_{m, m+1}=\alpha\left(N_{\max }-m\right) e^{-\beta m}$

c

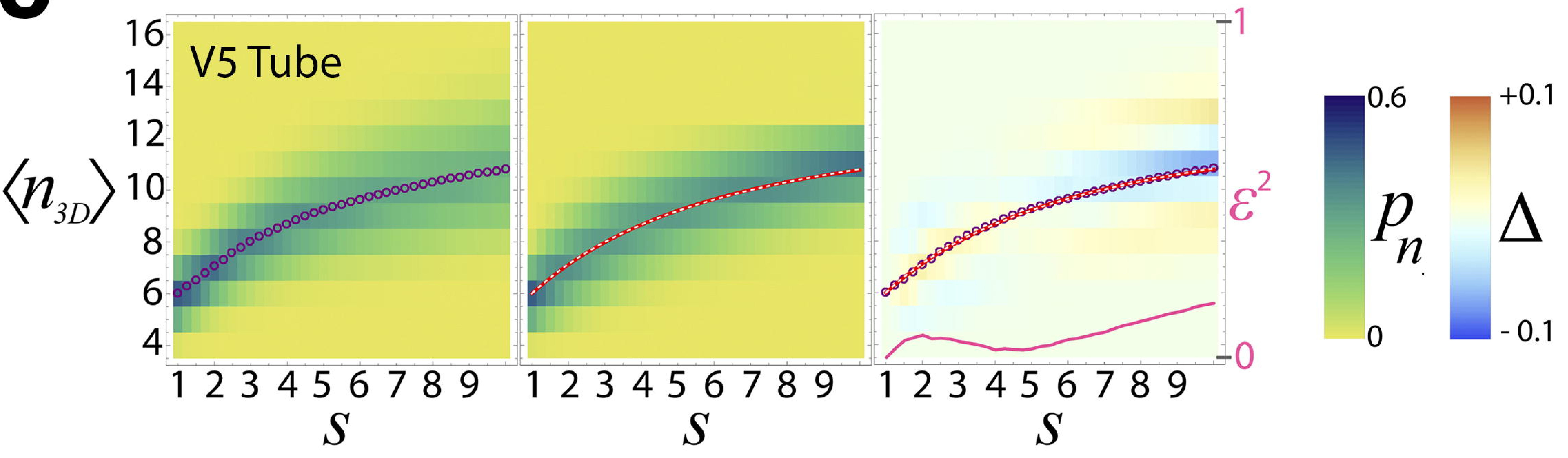



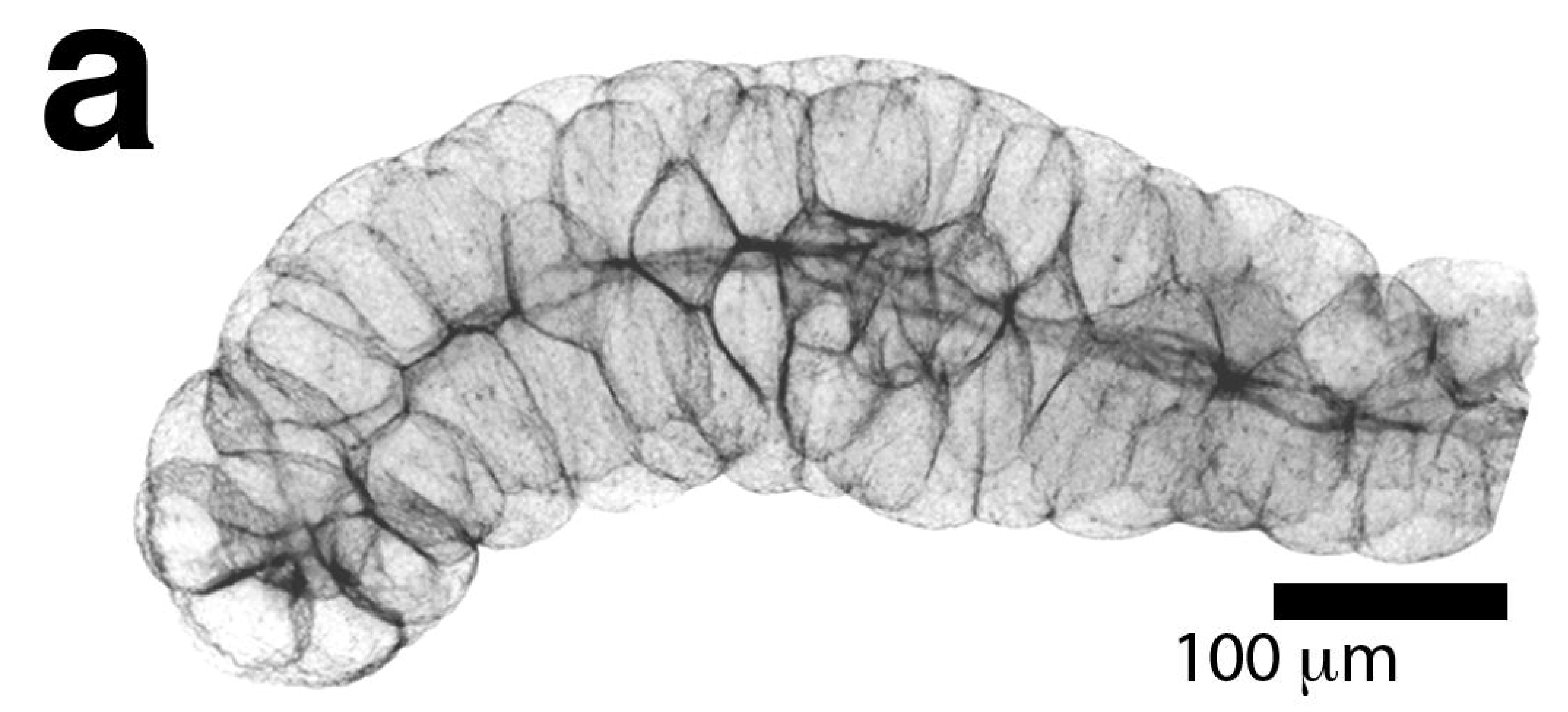

b

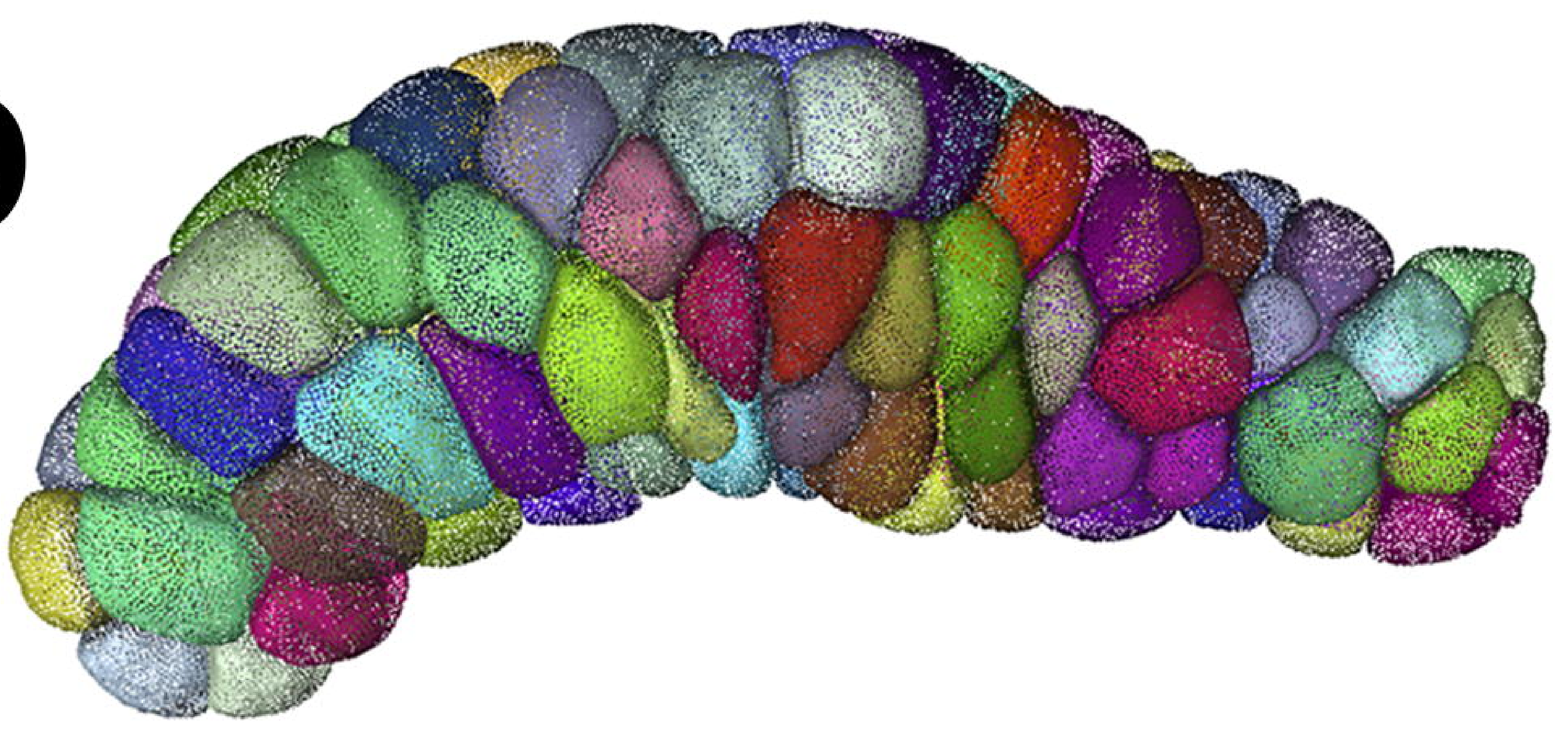

C salivary gland

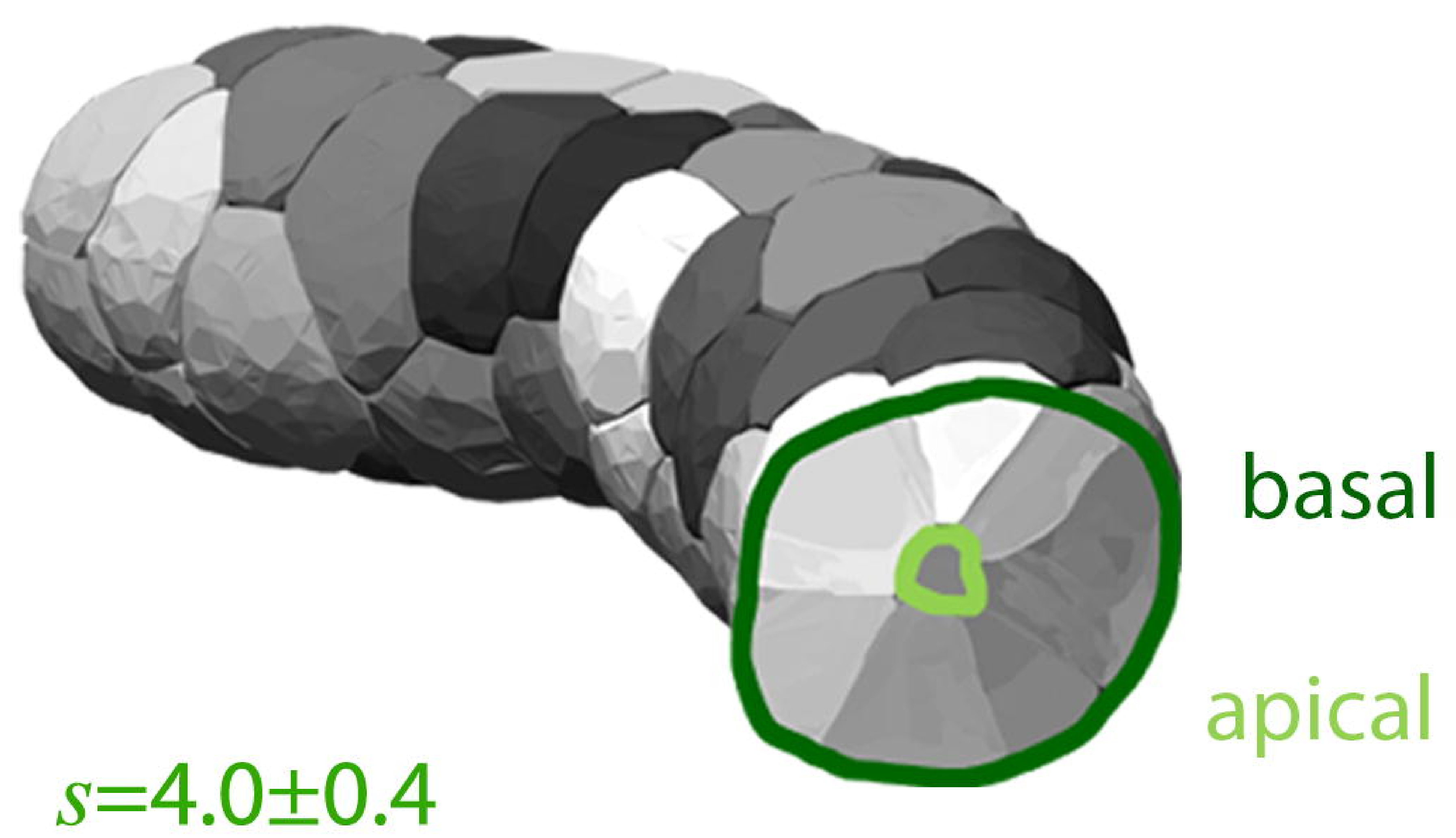

$\%$ scutoids $=76 \pm 11 \%$

d
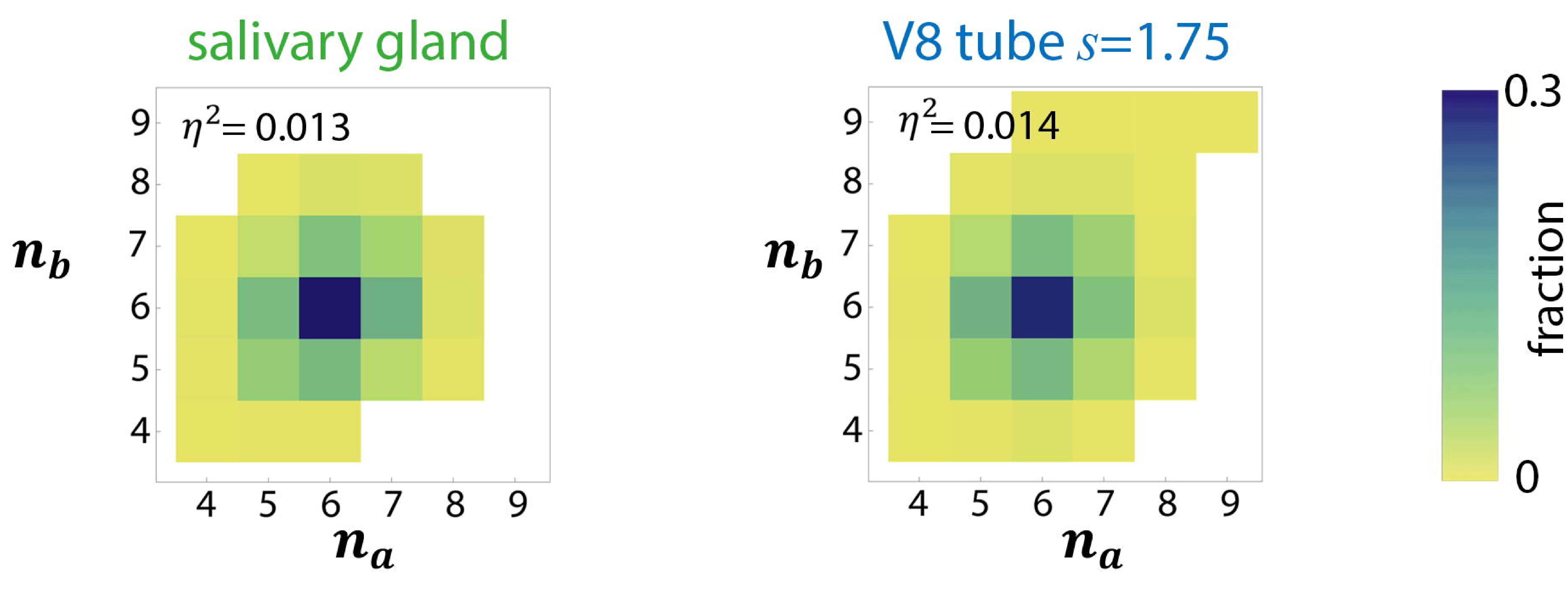

e

salivary gland
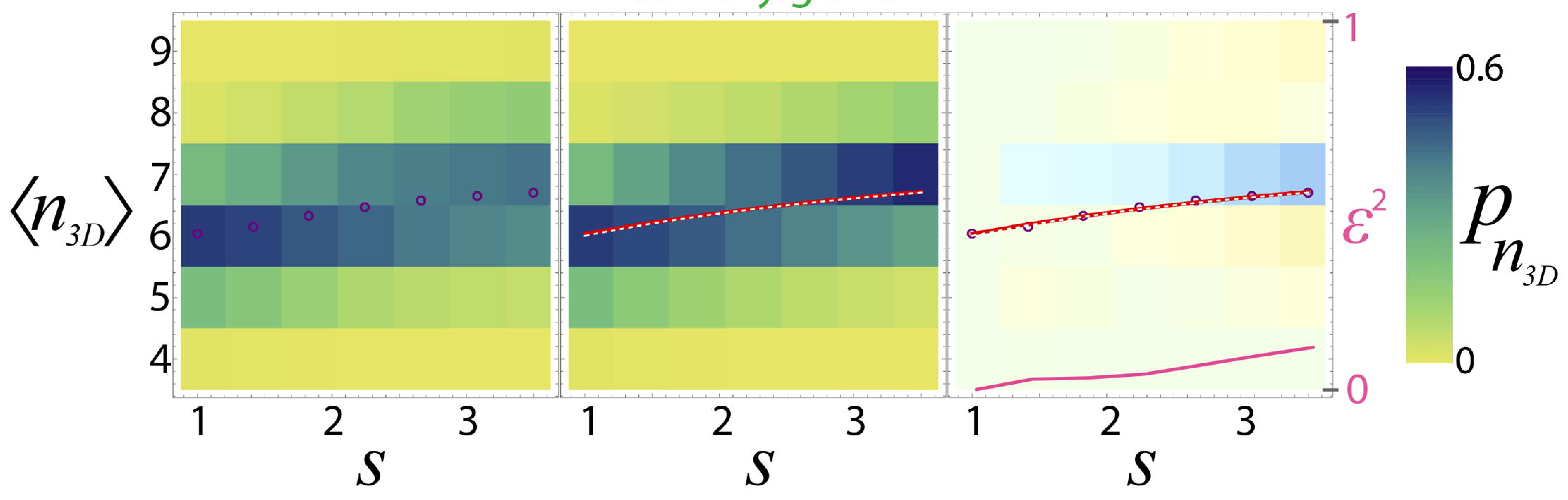

V8 tube

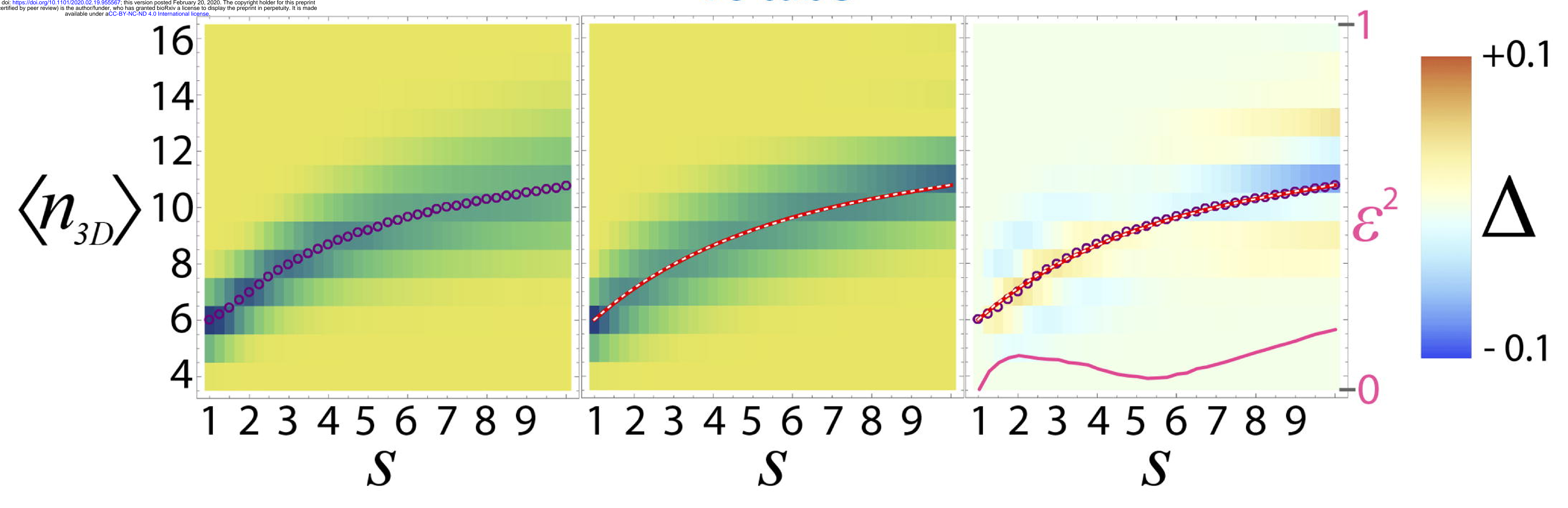

